\title{
Chinese Herbal Medicine Used With or Without Conventional Western Therapy for COVID-19: An Evidence Review of Clinical Studies
}

\begin{abstract}
Shi-Bing Liang ${ }^{1}$, Ying-Ying Zhang ${ }^{1}$, Chen Shen ${ }^{1}$, Chang-Hao Liang ${ }^{1}$, Bao-Yong Lai ${ }^{2}$, Ning Dai ${ }^{1}$, Yu-Qi Li ${ }^{1}$, Zi-Yu Tian ${ }^{1}$, Xiao-Wen Zhang ${ }^{1}$, Yue Jiang ${ }^{1}$, Min Xiong ${ }^{1}$, Ya-Peng Zhang ${ }^{1}$, Ying Zhang ${ }^{1}$, Nicola Robinson ${ }^{1,3}$ and Jian-Ping Liu ${ }^{1,4 *}$

${ }^{1}$ Centre for Evidence-Based Chinese Medicine, Beijing University of Chinese Medicine, Beijing, China, ${ }^{2}$ The Third Affiliated Hospital, Beijing University of Chinese Medicine, Beijing, China, ${ }^{3}$ School of Health and Social Care, London South Bank University, London, United Kingdom, ${ }^{4}$ Institute of Integrated Traditional Chinese Medicine and Western Medicine, Guangzhou Medical University, Guangzhou, China
\end{abstract}

OPEN ACCESS

Edited by:

Jia-bo Wang,

Fifth Medical Center of the PLA

General Hospital, China

Reviewed by:

Vernice Cheryl Nikodem,

University of Fort Hare, South Africa

Javad Sharifi-Rad,

Shahid Beheshti University of Medical Sciences, Iran

*Correspondence: Jian-Ping Liu

Liujp@bucm.edu.cn

Specialty section: This article was submitted to

Ethnopharmacology,

a section of the journal

Frontiers in Pharmacology

Received: 15 July 2020 Accepted: 10 December 2020 Published: 26 February 2021

Citation:

Liang S-B, Zhang $Y-Y$, Shen $C$, Liang C-H, Lai B-Y, Dai N, Li Y-Q,

Tian $Z-Y$, Zhang $X-W$, Jiang $Y$, Xiong $M$, Zhang $Y-P$, Zhang $Y$,

Robinson N and Liu J-P (2021)

Chinese Herbal Medicine Used With or

Without Conventional Western

Therapy for COVID-19: An Evidence

Review of Clinical Studies.

Front. Pharmacol. 11:583450.

doi: 10.3389/fphar.2020.583450
Objective: To present the evidence of the therapeutic effects and safety of Chinese herbal medicine (CHM) used with or without conventional western therapy for COVID-19.

Methods: Clinical studies on the therapeutic effects and safety of CHM for COVID-19 were included. We summarized the general characteristics of included studies, evaluated methodological quality of randomized controlled trials (RCTs) using the Cochrane risk of bias tool, analyzed the use of $\mathrm{CHM}$, used Revman 5.4 software to present the risk ratio $(\mathrm{RR})$ or mean difference (MD) and their 95\% confidence interval $(\mathrm{Cl})$ to estimate the therapeutic effects and safety of CHM.

Results: A total of 58 clinical studies were identified including RCTs $(17.24 \%, 10)$, nonrandomized controlled trials $(1.72 \%, 1)$, retrospective studies with a control group $(18.97 \%, 11)$, case-series $(20.69 \%, 12)$ and case-reports $(41.38 \%, 24)$. No RCTs of high methodological quality were identified. The most frequently tested oral Chinese patent medicine, Chinese herbal medicine injection or prescribed herbal decoction were: Lianhua Qingwen granule/capsule, Xuebijing injection and Maxing Shigan Tang. In terms of aggravation rate, pooled analyses showed that there were statistical differences between the intervention group and the comparator group (RR $0.42,95 \% \mathrm{Cl} 0.21$ to 0.82 , six RCTs; RR $0.38,95 \% \mathrm{Cl} 0.23$ to 0.64 , five retrospective studies with a control group), that is, $\mathrm{CHM}$ plus conventional western therapy appeared better than conventional western therapy alone in reducing aggravation rate. In addition, compared with conventional western therapy, CHM plus conventional western therapy had potential advantages in increasing the recovery rate and shortening the duration of fever, cough and fatigue, improving the negative conversion rate of nucleic acid test, and increasing the improvement rate of chest CT manifestations and shortening the time from receiving the treatment to the beginning of chest CT manifestations improvement. For adverse events, pooled data showed that there were no statistical differences between the $\mathrm{CHM}$ and the control groups. 
Conclusion: Current low certainty evidence suggests that there maybe a tendency that $\mathrm{CHM}$ plus conventional western therapy is superior to conventional western therapy alone. The use of $\mathrm{CHM}$ did not increase the risk of adverse events.

Keywords: traditional Chinese medicine, Chinese herbal medicine, novel coronavirus pneumonia, coronavirus disease 2019, COVID-19, SARS-CoV-2, review, clinical study

\section{INTRODUCTION}

Novel coronavirus pneumonia (NCP), officially named as Coronavirus Disease 2019 (COVID-19) by the World Health Organization (WHO) (World Health Organization, 2020a), is an acute respiratory infectious disease caused by severe acute respiratory syndrome coronavirus 2 (SARS-Cov-2) which has affected the general population. The main symptoms of COVID19 are fever, cough and fatigue, and may be accompanied by nasal congestion, runny nose, sore throat, diarrhea, or loss of taste and smell anosmia (National Health Commission of the People's Republic of China, 2020a). In traditional Chinese medicine, COVID-19 is classified within the pestilential (Yibing, 疫病) category. The National Health Commission of the People's Republic of China has incorporated COVID-19 into the category B infectious diseases as stipulated in the Law of the People's Republic of China on the Prevention and Control of Infectious Diseases, and carried out prevention and control management following category A infectious diseases. On 11 March 2020, the director-general of World Health Organization (WHO), Dr Tedros Adhanom Ghebreyesus, declared that COVID-19 was now characterized as a pandemic (World Health Organization, 2020b), that is, COVID-19 had spread worldwide, and posed a great challenge and threat to the existing public health resources.

At present, there is no specific and effective therapy for the treatment and prevention of this disease (Chandan et al., 2020; Torequl et al., 2020). Traditional Chinese medicine (TCM) has accumulated thousands of years of experience on the use of Chinese herbal medicine (CHM) to prevent and treat infectious diseases (Jiang 2011). Its success was initially substantiated by modern human clinical research on severe acute respiratory syndrome (SARS) and $\mathrm{H} 1 \mathrm{~N} 1$ influenza epidemics, suggesting that using historical CHM experience may be a worthwhile approach (Luo et al., 2020). As this current epidemic escalated into a pandemic, the National Health Commission of the People's Republic of China has released multiple editions of guidelines for the diagnosis and treatment of COVID-19 (hereinafter referred to as GDT of COVID-19). In the third edition (National Health Commission of the People's Republic of China, 2020b), CHM was recommended for the treatment of COVID-19, and all relevant medical institutions were required to actively encourage of the use of CHM in the treatment of COVID-19. The early application of CHM during the COVID-19 pandemic and appeared to have a potentially beneficial effects. CHM has increasingly shown its potential in the treatment and prevention for infectious diseases, and has received widespread attention.
To further probe the role of CHM used with or without conventional western therapy on the treatment of COVID-19, an evidence-based approach was employed to systematically collate, analyze and evaluate clinical studies on the therapeutic effects and safety of using CHM for COVID-19.

\section{MATERIALS AND METHODS}

\section{Inclusion and Exclusion Criteria of Studies}

The following criteria were used to identify relevant studies.

Inclusion criteria were as follows: 1) Clinical studies which aimed to evaluate the therapeutic effects and/or safety of CHM used with or without conventional western therapy in patients with COVID-19; 2) There were no limits on the study design and could be randomized controlled trials (RCT), non-randomized controlled trials (non-RCT), cohort studies, case series, case reports or other study designs; 3) Participants were patients diagnosed with COVID-19. Disease severity could be mild, common, severe or critical, as prescribed in the guideline for the diagnosis and treatment of COVID-19 formulated by the National Health Commission of the People's Republic of China. There was no limitation on participants' age, gender and their ethnicity, or the setting of the studies; 4) The interventions in the experimental group were $\mathrm{CHM}$ and included prescribed herbal decoctions, oral Chinese patent medicines (capsules, tablets or granules) or Chinese herbal medicine injection, or CHM combined with comparators. For controlled clinical studies, comparators could be conventional western therapy or placebo.

Exclusion criteria were: 1) The full text of the studies could not be obtained; 2) Any duplicated articles; 3) Registered clinical studies but had not started or completed; 4) Clinical studies that had been registered and completed but had not published research data, and the data which could not be obtained by contacting the authors; 5) If the registered protocol and the publication(s) were from the same study, the protocol was excluded.

\section{Retrieval Platforms and Search Strategies of Studies}

Studies were retrieved through nine electronic databases including: China National Knowledge Infrastructure (CNKI, as of April 30, 2020), Wanfang Database (from January 1 to April 30, 2020), the China Science Technology Journal Database (VIP, from January 1 to April 30, 2020), SinoMed (from January 1 to April 30, 2020), PubMed (from January 1 to April 30, 2020), Embase (from January 1 to April 30, 2020), BioRxiv (as of April 30, 2020), MedRxiv (as of April 30, 2020), arXiv (as of April 30, 2020) and clinical trial registration platforms (CTRPs) including 
ClinicalTrials.gov (www.clinicaltrials.gov, as of April 30, 2020) and Chinese Clinical Trial Registry (ChiCTR, www.chictr.org/cn, as of April 30, 2020).

For the databases/CTRPs with COVID-19 thematic platforms, including CNKI and ClinicalTrials.gov, the search was performed directly in the COVID-19 thematic platform. For Wanfang, VIP, SinoMed, PubMed and Embase, search terms were used. The search terms included Xinxing Guanzhuang Bingdu Bing (新型 冠状病毒病), Xinguan Feiyan (新冠肺炎), 2019 Guanzhuang Bingdu Bing (2019冠状病毒病), coronavirus disease-19, COVID-19, 2019 novel coronavirus, 2019-nCOV, NCP, Zhongyi (中医), Zhongyao (中药), Caoyao (草药), Tangji (汤 剂), Zhongchengyao (中成药), Zhusheji (注射剂), Zhongxiyi Jiehe (中西医结合), Chinese medicine, traditional Chinese medicine, herbal medicine, decoction, patent medicine, injection, integrated Chinese and western medicine. For ChiCTR, title search was carried out using Xinxing Guangzhuang Bingdu (新型冠状病毒) and COVID-19 as search terms. For BioRxiv, MedRxiv and arXiv, title or abstract search was carried out using COVID-19 as search terms. Appendix 1 shows the search strategies for the nine electronic databases and CTRP.

Before submission, we updated the search and included the latest published studies that met the inclusion criteria.

\section{Study Selection and Data Extraction}

Published studies were screened according to the inclusion/ exclusion criteria by titles, abstracts and (or) full texts of the published articles. Registered studies were screened according to the inclusion/exclusion criteria by reading the titles and details of registered protocols. SBL, YYZ, CS, CHL, YQL, BYL and ZYT were responsible for the selection of articles.

Excel 2010 was used to provide the data sheets for extraction. Extracted items include first author's name or registered protocol's ID, study titles, the country in which the study was carried out, study design, characteristics of participants (such as sample size, age, gender, severity of COVID-19, etc.), details of interventions and outcomes, etc. For each included study, two authors independently extracted and checked the data. The inconsistencies were resolved by the two authors through consultation. If any disagreements, a third author (JPL) was consulted. SBL, YYZ, YQL, CS, BYL, ND, YJ, XWZ, CHL, YPZ and MX participated in data extraction in pair.

\section{Outcomes}

Primary outcomes included cure rate, mortality rate and aggravation rate (the change in the disease severity category, or patients were admitted to the ICU, et al.).

Secondary outcomes included the recovery rate or the duration (time to recovery) of main symptoms (including fever, cough and fatigue), negative conversion rate of nucleic acid test for SARS-CoV-2, improvement or recovery of chest CT manifestations, length of hospitalization and adverse events.

For outcomes reported at multiple timepoints, we used the longest reported follow-up timepoint.

\section{Design of This Review and Data Synthesis}

This is an evidence review of clinical studies on the therapeutic effects and safety of CHM used with or without conventional western therapy for COVID-19. Initially, we summarized the general characteristics of the included studies and then the methodological quality of included RCTs was assessed by SBL and YQL using the Cochrane risk of bias tool (Higgins et al., 2011). Subsequently, counts and percentages were applied to analyze the use of CHM. Lastly, we evaluated the therapeutic effects and safety of CHM used with or without conventional western therapy for COVID-19. For studies without control group, such as case series and case reports, we only presented these findings qualitatively as they were not sufficient to probe the therapeutic effects of CHM for COVID-19 due to the absence of control and a high risk of bias in case selection. For studies with control group, we used Cochrane Collaboration Review Manager 5.4 (Revman 5.4) software to conduct meta-analysis of the data. We presented binary data as a risk ratio (RR) with its $95 \%$ confidence interval (CI), and continuous data as a mean difference (MD) with its 95\% CI. Considering potential sources of clinical heterogeneity, the random-effect model (REM) was used for meta-analysis. We planned to conduct the following subgroup analysis for the primary outcomes if data were available: 1) subgroup analysis based on the severity of COVID-19, to detect whether the therapeutic effects of CHM is related to the severity; 2) subgroup analysis based on the use of $\mathrm{CHM}$ with or without conventional western therapy, to detect whether CHM alone or whether CHM plus conventional western therapy is more beneficial for the treatment of COVID-19.

\section{RESULTS}

\section{Search Results}

Figure 1 shows the flow diagram for the searching and screening of published articles. A total of 4763 published articles were retrieved from the above-mentioned nine open electronic databases, of which 102 articles were selected by reading fulltexts and 54 were removed for various reasons. Finally, 48 published articles (representing 48 completed studies) met the inclusion criteria. Before submission, we updated the search and included 10 further completed studies that met the inclusion criteria. Figure 2 shows the flow diagram for searching and screening of registered clinical studies. A total of 1669 registered protocols were retrieved from the above-mentioned two CTRPs and 50 registered protocols (50 registered clinical studies) meeting the inclusion criteria. However, all the 50 registered studies were excluded due to their status as 'not yet started' or “in progress.”

Therefore, 58 published articles (representing 58 completed studies) were included in our review.

\section{The Characteristics of Included 58 Clinical Studies}

All the 58 clinical studies were conducted in China. Of these, 52 were published in Chinese and six were in English. Among the 


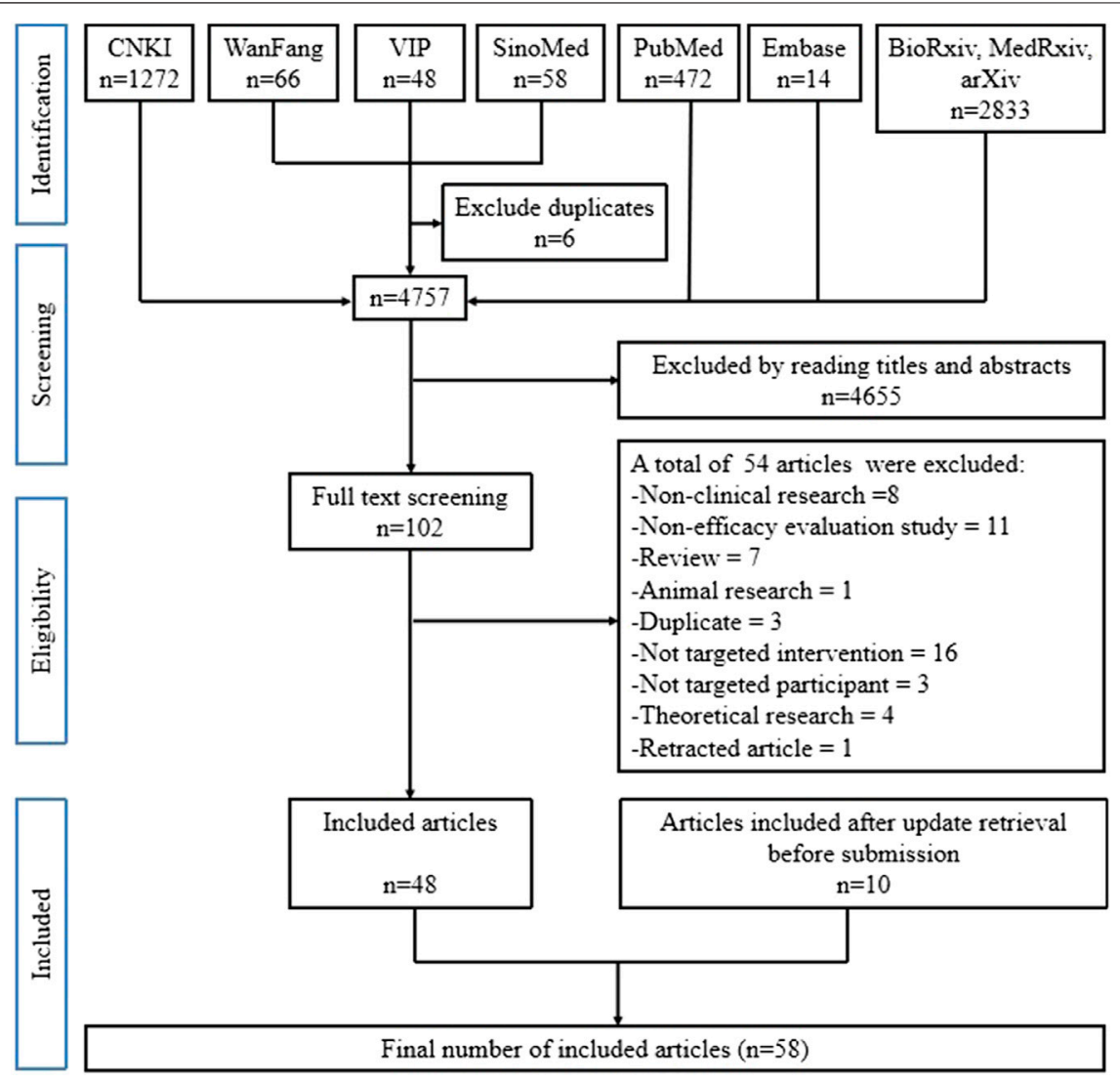

FIGURE 1 | Flow diagram for searching and screening of published articles.

included studies, 10 (17.24\%) were RCTs, one (1.72\%) was nonRCT, 11 (18.97\%) were retrospective studies with a control group, $12(20.69 \%)$ were case-series, 24 (41.38\%) were case-reports.

Of 2773 COVID-19 patients involved in the included studies, 1921 (69.28\%) received CHM. The level of severity of COVID-19 involved non-serious (including mild and common) and serious (including severe and critical). Of the included 58 studies, 29 (50.00\%) studies included only non-serious patients, 12 (20.69\%) studies included only serious patients, 11 (18.97\%) included both non-serious and serious patients, and the remaining $6(10.34 \%)$ studies did not report the level of severity of COVID-19.

Of the included 58 studies, 8 (13.79\%) involved only the use of $\mathrm{CHM}$, and 51 (87.93\%) involved CHM used in combination with conventional western therapy (such as abidor, ganciclovir, lopinavir, oxygen inhalation, nutritional support, etc.). The course of treatment varied from 4 to 15 days.

Table 1 shows the characteristics of the 58 included studies.

\section{Methodological Quality of RCTs}

In terms of the random sequence generation methods of the included 10 RCTs, six RCTs (Fu et al., 2020a; Wang et al., 2020c; Duan et al., 2020; Qiu et al., 2020; Sun et al., 2020; Yu et al., 2020) used random number tables, two trials (Ding et al., 2020; Ye, 2020) used a simple random allocation method and the remaining two RCTs (Zhang et al., 2020a; Fu et al., 2020b) only mentioned random without describing the detailed randomization method. Two RCTs (Wang et al., 2020c; Ye, 2020) performed allocation concealment. Therefore, the risk of selection (allocation) bias was unclear for the majority of the included RCTs due to lack of information on allocation concealment. Due to no trials used blinding to participants and personnel, the performance bias of all the included trials was judged as high-risk. Two RCTs (Wang et al., 2020c; Ye, 2020) performed outcome assessor blinding and the remaining eight RCTs (Fu et al., 2020a; Zhang et al., 2020a; Fu et al., 2020b; Ding et al., 2020; Duan et al., 2020; Qiu et al., 2020; Sun et al., 2020; Yu et al., 2020) did not report relevant information, thus the detection bias for the majority of the included RCTs was judged as unclear-risk. In terms of attrition bias, eight RCTs (Fu et al., 2020a; Zhang et al., 2020a; Wang et al., 2020c; Ding et al., 2020; Duan et al., 2020; Qiu et al., 2020; Sun et al., 2020; Ye, 2020) were assessed as low-risk of bias due to complete outcome data or incomplete outcome data being adequately addressed, two RCTs (Fu et al., 2020b; Yu et al., 2020) were assessed as high-risk due to incomplete outcome data that were not adequately addressed. Two RCTs (Wang et al., 2020c; Ye, 2020) registered the study protocol and reported the registration information. By comparison, we found that there was no selective reporting of outcomes in these two RCTs, so their reporting bias was evaluated as low-risk. Since the protocols or registration information of the other eight included RCTs (Yu et al., 2020; Duan et al., 2020; Sun et al., 


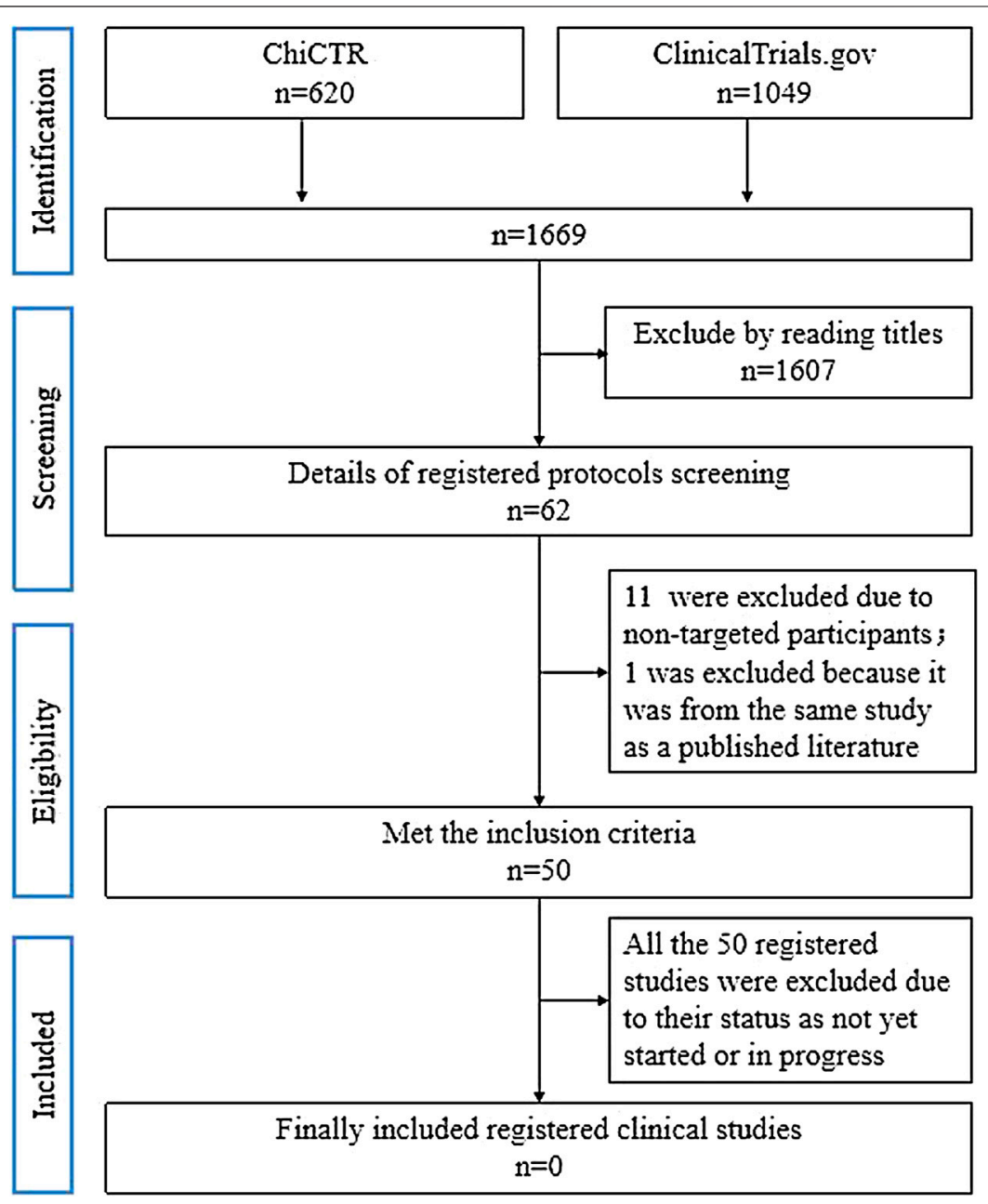

FIGURE 2 | Flow diagram for searching and screening of registered clinical studies.

2020; Fu et al., 2020a; Fu, et al., 2020b; Ding et al., 2020; Qiu et al., 2020; Zhang et al., 2020a) were not available, the selective reporting of outcomes in these RCTs could not be judged and the reporting bias of these was assessed as unclear-risk. All 10 RCTs reported the comparability of baseline data, so they were assessed as having a low-risk of other bias.

Figure 3 demonstrates the risk of bias of included 10 RCTs.

\section{Analysis of the use of CHM}

For the type of CHM, $24(41.38 \%)$ studies tested oral Chinese patent medicine, $40(68.97 \%)$ studies tested prescribed herbal decoction, and 7 (12.07\%) studies tested Chinese herbal medicine injection.

The top ten CHMs used were Maxing Shigan Tang [麻杏石甘 汤, 15.52\% (9/58)], Lianhua Qingwen granule/capsule [连花清瘟 颗粒/胶囊, $15.52 \%$ (9/58)], Xuebijing injection [血必净注射剂, 8.62\% (5/58)], Dayuanyin [达原饮, 8.62\% (5/58)], Shufeng Jiedu capsule[疏风解毒胶囊, 8.62\% (5/58)], Qingfei Paidu Tang [清肺 排毒汤, 6.90\% (4/58)], Xiaochaihu Tang [小柴胡汤, 6.90\% (4/ 58)], Ganlu Xiaodu Dan [甘露消毒丹, 5.17\% (3/58)], Liujunzi Tang [六君子汤, 5.17\% (3/58)] and Toujie Quwen granule [透解
袪瘟颗粒, 5.17\% (3/58)]. Of which, the most frequently used oral Chinese patent medicine, Chinese herbal medicine injection and prescribed herbal decoction were Lianhua Qingwen granule/ capsule [连花清瘟颗粒/胶囊], Xuebijing injection [血必净注 射剂], and Maxing Shigan Tang [麻杏石甘汤], respectively.

Table 2 lists the CHM used at least twice.

\section{Therapeutic effects and Safety of CHM in the Treatment or Adjuvant Treatment of COVID-19}

Analysis for Studies with Control Group

Primary Outcomes

Cure Rate. Six studies including one RCT (Fu et al., 2020b) and five retrospective studies with a control group (Qu et al., 2020a; Li et al., 2020c; Yang et al., 2020c; Xia et al., 2020; Shi et al., 2020a) reported this outcome. Of which, one study (Shi et al., 2020) was not enrolled into the meta-analysis due to no assessment criteria of cure rate in it's publication. All the other five studies adopted the judgment criteria of the GDT of COVID-19 for cure: 1) the body temperature returned to normal for longer than three days; 2) the respiratory symptoms 
TABLE 1 | The characteristics of included studies of Chinese herbal medicine for COVID-19.

\begin{tabular}{|c|c|c|c|c|c|c|c|c|}
\hline Study ID & Sample size (M/F) & Age (year) & $\begin{array}{c}\text { The severity }\left(^{\star}\right) \text { of } \\
\text { COVID-19 }\end{array}$ & $\begin{array}{l}\text { Type of Chinese } \\
\text { herbal medicine }\end{array}$ & $\begin{array}{c}\text { Conventional } \\
\text { western therapy } \\
\text { (Yes/No) }\end{array}$ & $\begin{array}{l}\text { Course of } \\
\text { CHM } \\
\text { treatment }\end{array}$ & Outcomes & $\begin{array}{l}\text { Author's conclusion } \\
\text { towards the role of } \\
\text { Chinese herbal medicine } \\
\text { in the treatment or } \\
\text { adjuvant treatment of } \\
\text { COVID-19 (positive/ } \\
\text { negative) }\end{array}$ \\
\hline \multicolumn{9}{|c|}{ Study type 1 : randomized controlled trials $(10,17.24 \%)$} \\
\hline Yu et al. (2020) & T:82/65, C:89/59 & $\begin{array}{l}\mathrm{T}: 48.27 \pm 9.56, \mathrm{C}: \\
47.25 \pm 8.67\end{array}$ & Non-serious & $\begin{array}{l}\text { Chinese patent } \\
\text { medicine }\end{array}$ & Yes & 7 days & (2) (3) (11) (13) & Positive \\
\hline Duan et al. (2020) & $\mathrm{T}: 39 / 43, \mathrm{C}: 23 / 18$ & $\begin{array}{l}\text { T: } 51.99 \pm 13.88, \mathrm{C}: \\
50.29 \pm 13.17\end{array}$ & Non-serious & $\begin{array}{l}\text { Chinese patent } \\
\text { medicine }\end{array}$ & Yes & 5 days & (2) (4) (5) (6) (13) & Positive \\
\hline Sun et al. (2020) & T:17/15, C:11/14 & $\begin{array}{l}\mathrm{T}: 45.4 \pm 14.10, \mathrm{C}: \\
42.0 \pm 11.70\end{array}$ & Non-serious & $\begin{array}{l}\text { Chinese patent } \\
\text { medicine }\end{array}$ & Yes & 14 days & (2) (4) (5) (6) (8) (1) & Positive \\
\hline Fu et al. (2020a) & T:17/15, C:19/14 & $\begin{array}{l}\mathrm{T}: 43.26 \pm 7.15, \mathrm{C}: \\
43.68 \pm 6.45\end{array}$ & Non-serious & $\begin{array}{l}\text { Chinese patent } \\
\text { medicine }\end{array}$ & Yes & 10 days & (2) (11) (13) & Positive \\
\hline Fu et al. (2020b) & T:19/18, C:19/17 & $\begin{array}{l}\mathrm{T}: 45.26 \pm 7.25, \mathrm{C}: \\
44.68 \pm 7.45\end{array}$ & Non-serious & $\begin{array}{l}\text { Chinese patent } \\
\text { medicine }\end{array}$ & Yes & 15 days & (1) (2) (13) & Positive \\
\hline Ding et al. (2020) & T:39/12, C:39/10 & $\begin{array}{l}\mathrm{T}: 54.7 \pm 21.3, \mathrm{C}: \\
50.8 \pm 23.5\end{array}$ & $\begin{array}{l}\text { T: } 46 \text { (non-serious) / } 5 \\
\text { (serious), C: } 11 \text { (non- } \\
\text { serious) / } 4 \text { (serious) }\end{array}$ & $\begin{array}{l}\text { Prescribed herbal } \\
\text { decoction }\end{array}$ & Yes & 10 days & (4) (5) (11) (13) & Positive \\
\hline Ye (2020) & $\mathrm{T}: 2 / 26, \mathrm{C}: 4 / 10$ & T:53.5-69, C:47-67 & Serious & $\begin{array}{l}\text { Prescribed herbal } \\
\text { decoction }\end{array}$ & Yes & 7 days & (2) (3) & Positive \\
\hline Qiu et al. (2020) & $\mathrm{T}: 13 / 12, \mathrm{C}: 14 / 11$ & $\begin{array}{l}\text { T: } 53.35 \pm 18.35, \mathrm{C}: \\
51.32 \pm 14.62\end{array}$ & Non-serious & $\begin{array}{l}\text { Prescribed herbal } \\
\text { decoction }\end{array}$ & Yes & 10 days & (2) (7) (8) (11) & Positive \\
\hline Zhang et al. (2020a) & $\mathrm{T}: 9 / 13, \mathrm{C}: 10 / 13$ & $\begin{array}{l}\mathrm{T}: 53.7 \pm 3.5, \mathrm{C}: \\
55.6 \pm 4.2\end{array}$ & Non-serious & $\begin{array}{l}\text { Prescribed herbal } \\
\text { decoction }\end{array}$ & Yes & 7 days & (7) (8) (9) (11) (13) & Positive \\
\hline Wang et al. (2020c) & T:14/10, C:12/11 & $\begin{array}{l}\mathrm{T}: 46.8 \pm 14.4, \mathrm{C}: \\
51.4 \pm 17.6\end{array}$ & Non-serious & $\begin{array}{l}\text { Prescribed herbal } \\
\text { decoction }\end{array}$ & Yes & 14 days & (2) (3) (7) (13) & Positive \\
\hline \multicolumn{9}{|c|}{ Study type 2: Non-randomized controlled trial (1, 1.72\%) } \\
\hline Xiao et al. (2020) & $\mathrm{T}: 64 / 36, \mathrm{C}: 66 / 34$ & $\begin{array}{l}\mathrm{T}: 60.90 \pm 8.70, \mathrm{C}: \\
62.20 \pm 7.50\end{array}$ & Non-serious & $\begin{array}{l}\text { Chinese patent } \\
\text { medicine }\end{array}$ & Yes & 14 days & (7) (8) (9) (11) (13) & Positive \\
\hline \multicolumn{9}{|c|}{ Study type 3: Retrospective studies with a control group $(11,18.97 \%)$} \\
\hline Cheng et al. (2020) & $\mathrm{T}: 26 / 25, \mathrm{C}: 27 / 24$ & $\begin{array}{l}\mathrm{T}: 55.5 \pm 12.3, \mathrm{C}: \\
55.8 \pm 11.6\end{array}$ & Non-serious & $\begin{array}{l}\text { Chinese patent } \\
\text { medicine }\end{array}$ & Yes & 7 days & (2) (4)(5)(6)(7)(8)(9) (11) & Positive \\
\hline Liu et al. (2020c) & $\mathrm{T}: 21 / 23, \mathrm{C}: 16 / 20$ & $\mathrm{~T}: 50.73, \mathrm{C}: 51.75$ & $\begin{array}{l}\text { T: } 37 \text { (non-serious) / } 7 \\
\text { (serious), C: } 28 \text { (non- } \\
\text { serious) / } 8 \text { (serious) }\end{array}$ & $\begin{array}{l}\text { Chinese patent } \\
\text { medicine }\end{array}$ & Yes & 7 days & (11) (13) & Positive \\
\hline Zhang et al. (2020b) & $\mathrm{T}: 10 / 12, \mathrm{C}: 12 / 10$ & $\mathrm{~T}: 25-73, \mathrm{C}: 19-67$ & Non-serious & $\begin{array}{l}\text { Chinese herbal } \\
\text { medicine injection }\end{array}$ & Yes & 7 days & (10) (11) (13) & Positive \\
\hline Li et al. (2020c) & $\mathrm{T}: 15 / 15, \mathrm{C}: 13 / 17$ & $\begin{array}{l}\mathrm{T}: 53.600 \pm 0.259, \mathrm{C}: \\
50.433 \pm 0.338\end{array}$ & $\begin{array}{l}\text { T: } 3 \text { (serious)/27(not } \\
\text { reported) , C: } 2 \\
\text { (serious)/28(not } \\
\text { reported) }\end{array}$ & $\begin{array}{l}\text { Prescribed herbal } \\
\text { decoction }\end{array}$ & Yes & Not reported & (1) (2) (7) (8) (1) (13) & Positive \\
\hline Yang et al. (2020c) & T:28/23, C: $24 / 28$ & $\begin{array}{l}\mathrm{T}: 61.57 \pm 1.84, \mathrm{C}: \\
66.35 \pm 1.82\end{array}$ & Serious & $\begin{array}{l}\text { Prescribed herbal } \\
\text { decoction + Chinese } \\
\text { herbal medicine } \\
\text { injection }\end{array}$ & Yes & Not reported & (1) (3) (11) (12) (13) & Positive \\
\hline Qu et al. (2020a) & T:25/15, C:16/14 & $\begin{array}{l}\mathrm{T}: 40.65 \pm 8.23, \mathrm{C}: \\
39.82 \pm 6.40\end{array}$ & Non-serious & $\begin{array}{l}\text { Chinese patent } \\
\text { medicine }\end{array}$ & Yes & 10 days & (1) (7) (8) (9) (10) (3) & Positive \\
\hline
\end{tabular}


TABLE 1 | (Continued) The characteristics of included studies of Chinese herbal medicine for COVID-19.

\begin{tabular}{|c|c|c|c|c|c|c|c|c|}
\hline Study ID & Sample size (M/F) & Age (year) & $\begin{array}{l}\text { The severity }\left(^{*}\right) \text { of } \\
\text { COVID-19 }\end{array}$ & $\begin{array}{l}\text { Type of Chinese } \\
\text { herbal medicine }\end{array}$ & $\begin{array}{l}\text { Conventional } \\
\text { western therapy } \\
\text { (Yes/No) }\end{array}$ & $\begin{array}{l}\text { Course of } \\
\text { CHM } \\
\text { treatment }\end{array}$ & Outcomes & $\begin{array}{l}\text { Author's conclusion } \\
\text { towards the role of } \\
\text { Chinese herbal medicine } \\
\text { in the treatment or } \\
\text { adjuvant treatment of } \\
\text { covID-19 (positive/ } \\
\text { negative) }\end{array}$ \\
\hline Xia et al. (2020) & $\mathrm{T}: 17 / 17, \mathrm{C}: 6 / 12$ & $\begin{array}{l}\text { T:54.18 } \pm 13.08, \mathrm{C}: \\
53.67 \pm 12.70\end{array}$ & $\begin{array}{l}\text { T: } 27 \text { (non-serious) / } 7 \\
\text { (serious) , C: } 13 \text { (non- } \\
\text { serious) / } 4 \text { (serious) }\end{array}$ & $\begin{array}{l}\text { Chinese patent } \\
\text { medicine + Chinese } \\
\text { herbal medicine } \\
\text { injection + prescribed } \\
\text { herbal decoction }\end{array}$ & Yes & $5-10$ days & (1) (2) (3) (7) (11) (12) (13) & Positive \\
\hline Yao et al. (2020) & $\mathrm{T}: 16 / 5, \mathrm{C}: 12 / 9$ & $\begin{array}{l}\text { T:57.1 } 14.0, \mathrm{C}: \\
62.4 \pm 12.3\end{array}$ & Non-serious & $\begin{array}{l}\text { Chinese patent } \\
\text { medicine }\end{array}$ & Yes & Not reported & (4) (5) (6) (7) & Positive \\
\hline Shi et al. (2020a) & $\mathrm{T}: 26 / 23, \mathrm{C}: 10 / 8$ & $\begin{array}{l}\mathrm{T}: 47.94 \pm 14.46, \mathrm{C}: \\
46.72 \pm 17.40\end{array}$ & $\begin{array}{l}\text { T: } 41 \text { (non-serious) / } 8 \\
\text { (serious) , C: } 15 \text { (non- } \\
\text { serious) / } 3 \text { (serious) }\end{array}$ & $\begin{array}{l}\text { Chinese patent } \\
\text { medicine + prescribed } \\
\text { herbal decoction }\end{array}$ & Yes & Not reported & (1) (2) (11) (12) & Positive \\
\hline Yang et al. (2020a) & T:16/10, C:9/14 & $\begin{array}{l}\mathrm{T}: 50.35 \pm 13.37, \mathrm{C:} \\
47.17 \pm 16.57\end{array}$ & Non-serious & $\begin{array}{l}\text { Chinese patent } \\
\text { medicine }\end{array}$ & Yes & 7 days & (2) (10) (11) (13) & Positive \\
\hline Chen et al. (2020) & T:14/20, C:15/19 & $\begin{array}{l}\text { T:65.06 } \pm 10.63, \mathrm{C}: \\
64.35 \pm 10.34\end{array}$ & Non-serious & $\begin{array}{l}\text { Chinese patent } \\
\text { medicine }\end{array}$ & Yes & 7 days & (2) (4) (5) (6) (7) (8) (9) (11) (12) (13) & Positive \\
\hline \multicolumn{9}{|c|}{ Study type 4: Case-series (12, 20.69\%) } \\
\hline Zhang et al. (2020c) & 9/15 & $49.96 \pm 12.79(27-69)$ & Non-serious & $\begin{array}{l}\text { Prescribed herbal } \\
\text { decoction }\end{array}$ & Yes & $6-14$ days & NA & Positive \\
\hline Wang et al. (2020d) & $52 / 46$ & $42.70 \pm 16.86$ & $\begin{array}{l}87 \text { (non-serious) / } 11 \\
\text { (serious) }\end{array}$ & $\begin{array}{l}\text { Prescribed herbal } \\
\text { decoction }\end{array}$ & No & 9 days & NA & Positive \\
\hline Xie et al. (2020a) & 8 & $35-79$ & Serious & $\begin{array}{l}\text { Prescribed herbal } \\
\text { decoction }\end{array}$ & Yes & Not reported & NA & Positive \\
\hline Li et al. (2020d) & $3 / 3$ & $42-79$ & Serious & $\begin{array}{l}\text { Chinese patent } \\
\text { medicine + Chinese } \\
\text { herbal medicine } \\
\text { injection + prescribed } \\
\text { herbal decoction }\end{array}$ & Yes & Not reported & NA & Positive \\
\hline Ba et al. (2020) & $243 / 208$ & $43-66$ & $\begin{array}{l}399 \text { (non-serious) / } 46 \\
\text { (serious) }\end{array}$ & $\begin{array}{l}\text { Prescribed herbal } \\
\text { decoction }\end{array}$ & Yes & Not reported & NA & Positive \\
\hline Liu et al. (2020a) & 36 & $N R$ & Not reported & $\begin{array}{l}\text { Prescribed herbal } \\
\text { decoction }\end{array}$ & Yes & 14 days & NA & Positive \\
\hline Huang et al. (2020) & $38 / 33$ & $41.3 \pm 16.7$ & Non-serious & $\begin{array}{l}\text { Chinese patent } \\
\text { medicine + Chinese } \\
\text { herbal medicine } \\
\text { injection + prescribed } \\
\text { herbal decoction }\end{array}$ & Yes & Not reported & NA & Positive \\
\hline Xie et al. (2020b) & 27 & $2-68$ & Non-serious & $\begin{array}{l}\text { Prescribed herbal } \\
\text { decoction }\end{array}$ & Yes & Not reported & NA & Positive \\
\hline Cheng and Li (2020) & $29 / 25$ & $60.1 \pm 16.98(25-95)$ & Non-serious & $\begin{array}{l}\text { Chinese patent } \\
\text { medicine }\end{array}$ & Yes & $\begin{array}{l}\text { 8. } 0 \pm 4.10 \\
\text { days }\end{array}$ & NA & Positive \\
\hline Zhou et al. (2020) & $17 / 23$ & $19-68$ & Non-serious & $\begin{array}{l}\text { Prescribed herbal } \\
\text { decoction }\end{array}$ & Yes & 14 days & NA & Positive \\
\hline Qu et al. (2020b) & $23 / 17$ & $61.2 \pm 16.5(24-79)$ & Non-serious & $\begin{array}{l}\text { Prescribed herbal } \\
\text { decoction }\end{array}$ & Yes & 7 days & NA & $\begin{array}{l}\text { Positive } \\
\text { ontinued on following page) }\end{array}$ \\
\hline
\end{tabular}


TABLE 1 | (Continued) The characteristics of included studies of Chinese herbal medicine for COVID-19

\begin{tabular}{|c|c|c|c|c|c|c|c|c|}
\hline Study ID & Sample size (M/F) & Age (year) & $\begin{array}{l}\text { The severity }\left(^{*}\right) \text { of } \\
\text { COVID-19 }\end{array}$ & $\begin{array}{l}\text { Type of Chinese } \\
\text { herbal medicine }\end{array}$ & $\begin{array}{l}\text { Conventional } \\
\text { western therapy } \\
\text { (Yes/No) }\end{array}$ & $\begin{array}{l}\text { Course of } \\
\text { CHM } \\
\text { treatment }\end{array}$ & Outcomes & $\begin{array}{l}\text { Author's conclusion } \\
\text { towards the role of } \\
\text { Chinese herbal medicine } \\
\text { in the treatment or } \\
\text { adjuvant treatment of } \\
\text { COVID-19 (positive/ } \\
\text { negative) }\end{array}$ \\
\hline Shi et al. (2020c) & $15 / 25$ & $43.9 \pm 16.3(20-94)$ & $\begin{array}{l}32 \text { (non-serious) / } 8 \\
\text { (serious) }\end{array}$ & $\begin{array}{l}\text { Prescribed herbal } \\
\text { decoction }\end{array}$ & Yes & Not reported & NA & Positive \\
\hline \multicolumn{9}{|c|}{ Study type 5: Case-reports $(24,41.38 \%)$} \\
\hline Fu et al. (2020) & $1 / 1$ & 32,46 & Non-serious & $\begin{array}{l}\text { Chinese patent } \\
\text { medicine }\end{array}$ & Yes & $10 / 14$ days & NA & Positive \\
\hline Tian et al. (2020) & $2 / 3$ & $24,28,36,40,49$ & $\begin{array}{l}2 \text { (non-serious) / } 3 \\
\text { (serious) }\end{array}$ & $\begin{array}{l}\text { prescribed herbal } \\
\text { decoction + Chinese } \\
\text { patent medicine }\end{array}$ & Yes & 9 days & NA & Positive \\
\hline Dong et al. (2020) & $1 \mathrm{M}$ & 56 & Not reported & $\begin{array}{l}\text { Prescribed herbal } \\
\text { decoction }\end{array}$ & No & 11 day & NA & Positive \\
\hline Shi et al. (2020b) & $2 \mathrm{M}$ & 45,48 & Non-serious & $\begin{array}{l}\text { Prescribed herbal } \\
\text { decoction + Chinese } \\
\text { herbal medicine } \\
\text { injection }\end{array}$ & Yes & $7 / 18$ days & NA & Positive \\
\hline Li et al. (2020b) & $1 / 1$ & 35,36 & $\begin{array}{l}1 \text { (non-serious) / } 1 \\
\text { (serious) }\end{array}$ & $\begin{array}{l}\text { Prescribed herbal } \\
\text { decoction }\end{array}$ & No & 4/6 days & NA & Positive \\
\hline Zhao et al. (2020) & $1 \mathrm{~F}$ & 41 & Not reported & $\begin{array}{l}\text { Prescribed herbal } \\
\text { decoction }\end{array}$ & Yes & 9 days & NA & Positive \\
\hline He et al. (2020) & $2 \mathrm{M}$ & 25,29 & Serious & $\begin{array}{l}\text { Prescribed herbal } \\
\text { decoction }\end{array}$ & Yes & $8 / 6$ days & NA & Positive \\
\hline Yang and Niu (2020) & $1 \mathrm{~F}$ & 74 & Serious & $\begin{array}{l}\text { Prescribed herbal } \\
\text { decoction }\end{array}$ & Yes & 15 days & NA & Positive \\
\hline Wang et al. (2020e) & $2 \mathrm{M}$ & 33,54 & $\begin{array}{l}1 \text { (non-serious) / } 1 \\
\text { (serious) }\end{array}$ & $\begin{array}{l}\text { Prescribed herbal } \\
\text { decoction }\end{array}$ & Yes & Not reported & NA & Positive \\
\hline Li et al. (2020e) & $1 \mathrm{~F}$ & 71 & Serious & $\begin{array}{l}\text { Prescribed herbal } \\
\text { decoction }\end{array}$ & Yes & Not reported & NA & Positive \\
\hline Feng et al. (2020) & $1 \mathrm{~F}$ & 51 & Serious & $\begin{array}{l}\text { Prescribed herbal } \\
\text { decoction }\end{array}$ & Yes & 15 days & NA & Positive \\
\hline Xu et al. (2020) & $1 \mathrm{M}$ & 35 & Non-serious & $\begin{array}{l}\text { Prescribed herbal } \\
\text { decoction }\end{array}$ & Yes & 12 days & NA & Positive \\
\hline Liu et al. (2020b) & $1 \mathrm{~F}$ & 38 & Non-serious & $\begin{array}{l}\text { Prescribed herbal } \\
\text { decoction }\end{array}$ & Yes & 7 days & NA & Positive \\
\hline Li et al. (2020f) & $2 \mathrm{~F}$ & 17,45 & Non-serious & $\begin{array}{l}\text { Chinese patent } \\
\text { medicine + prescribed } \\
\text { herbal decoction }\end{array}$ & No & 9 days & NA & Positive \\
\hline Lin et al. (2020) & $1 \mathrm{~F}$ & 35 & Not reported & $\begin{array}{l}\text { Prescribed herbal } \\
\text { decoction }\end{array}$ & Yes & 12 days & NA & Positive \\
\hline Hu et al. (2020) & $1 \mathrm{~F}$ & 61 & Serious & $\begin{array}{l}\text { Chinese patent } \\
\text { medicine + prescribed } \\
\text { herbal decoction }\end{array}$ & Yes & 11 days & NA & Positive \\
\hline Wang et al. (2020f) & $3 / 1$ & $19,32,63,63$ & $\begin{array}{l}2 \text { (non-serious) / } 2 \\
\text { (serious) }\end{array}$ & $\begin{array}{l}\text { Chinese patent } \\
\text { medicine }\end{array}$ & Yes & Not reported & NA & Positive \\
\hline
\end{tabular}


TABLE 1 | (Continued) The characteristics of included studies of Chinese herbal medicine for COVID-19.

\begin{tabular}{|c|c|c|c|c|c|c|c|c|}
\hline Study ID & Sample size (M/F) & Age (year) & $\begin{array}{l}\text { The severity }\left(^{\star}\right) \text { of } \\
\text { COVID-19 }\end{array}$ & $\begin{array}{l}\text { Type of Chinese } \\
\text { herbal medicine }\end{array}$ & $\begin{array}{l}\text { Conventional } \\
\text { western therapy } \\
\text { (Yes/No) }\end{array}$ & $\begin{array}{l}\text { Course of } \\
\text { CHM } \\
\text { treatment }\end{array}$ & Outcomes & $\begin{array}{l}\text { Author's conclusion } \\
\text { towards the role of } \\
\text { Chinese herbal medicine } \\
\text { in the treatment or } \\
\text { adjuvant treatment of } \\
\text { COVID-19 (positive/ } \\
\text { negative) }\end{array}$ \\
\hline Deng et al. (2020) & $1 \mathrm{~F}$ & 39 & Serious & $\begin{array}{l}\text { Prescribed herbal } \\
\text { decoction }\end{array}$ & Yes & Not reported & NA & Positive \\
\hline Ni et al. (2020) & $1 / 2$ & $27,51,53$ & Serious & $\begin{array}{l}\text { Chinese patent } \\
\text { medicine }\end{array}$ & 1 Yes / 2 No & Not reported & NA & Positive \\
\hline Gao et al. (2020) & $1 F$ & 42 & Non-serious & $\begin{array}{l}\text { Chinese patent } \\
\text { medicine }\end{array}$ & No & 7 days & NA & Positive \\
\hline Li et al. (2020a) & $1 / 1$ & 68,47 & Non-serious & $\begin{array}{l}\text { Prescribed herbal } \\
\text { decoction }\end{array}$ & No & Not reported & NA & Positive \\
\hline Lai et al. (2020) & $1 / 2$ & $56,61,60$ & $\begin{array}{l}1 \text { (non-serious) / } 2 \text { (not } \\
\text { reported) }\end{array}$ & $\begin{array}{l}\text { Prescribed herbal } \\
\text { decoction }\end{array}$ & No & $6 / 7$ days & NA & Positive \\
\hline Wang et al. (2020a) & $1 / 1$ & 45,32 & Serious & $\begin{array}{l}\text { Chinese herbal } \\
\text { medicine injection + } \\
\text { prescribed herbal } \\
\text { decoction }\end{array}$ & Yes & 12/14days & NA & Positive \\
\hline Wang et al. (2020b) & $1 / 1$ & 63,49 & Non-serious & $\begin{array}{l}\text { Prescribed herbal } \\
\text { decoction }\end{array}$ & Yes & 10/14 days & NA & Positive \\
\hline
\end{tabular}

Note: $M$, male; $F$, female; T, treatment group involving Chinese herbal medicine; $C$, controlled group not involving Chinese herbal medicine; Yes, the intervention involved in this study was Chinese herbal medicine combined with conventional western therapy: No, the intervention involved in this trial was Chinese herbal medicines alone, not combined with conventional western therapy; NA, not applicable; Positive, Chinese herbal medicine has benefits on the treatment or adjuvant treatment of COVID-19; negative, Chinese herbal medicine has no benefits on the treatment or adjuvant treatment of COVID-19, or can even make the disease worse.

The severity (") was classified according to the guidelines for the diagnosis and treatment of COVID-19 released by the National Health Commission of the People's Republic of China. We divide them into two categories of non-serious (including mild and common) and serious (including severe and critical).

Although the article (Wang et al., 2020c) did not specify the severity of COVID-19, since all participants in this trial were screened from suspected COVID-19 patients, we considered the severity of COVID-19 of these participants as nonserious.

Outcomes: (1) cure rate; (2) aggravation rate; (3) mortality rate; (4) the recovery rate of fever; (5) the recovery rate of cough; (6) the recovery rate of fatigue; (7) the duration of fever; (8) the duration of cough; (9) the duration of fatigue; (1) negative conversion rate of nucleic acid test; (11) improvement or recovery of chest CT manifestations; (12) Length of hospitalization; (3) adverse events.

Although one trial (Yu et al., 2020) reported the outcome of aggravation rate, we did not enrolled the data on this outcome in the statistical analysis due to the inconsistency between the data presented in the table and in the text of the trial's publication. 


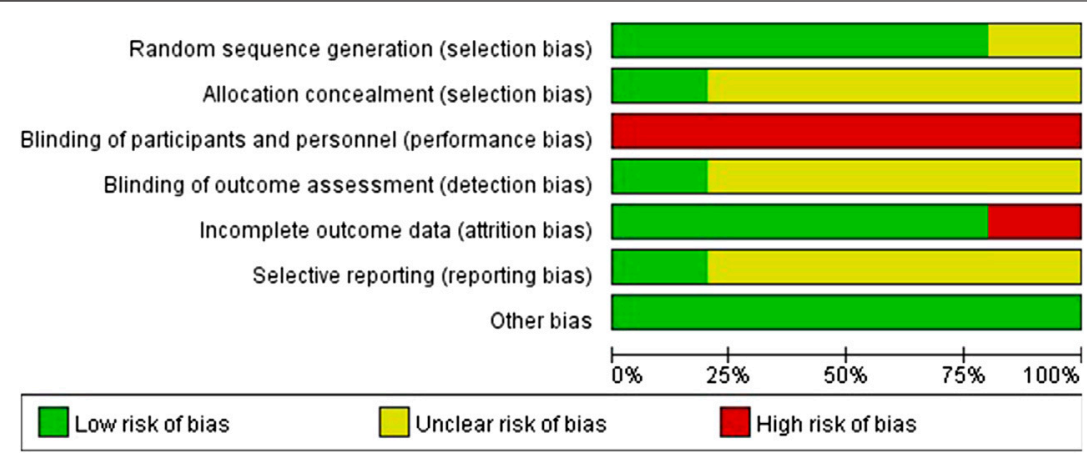

FIGURE 3 | Risk of bias graph of included 10 RCTs.

improved significantly; 3) the pulmonary imaging showed that the inflammation has obviously disappeared; 4) the respiratory pathogenic nucleic acid, (the sampling time interval of two tests was at least 1 day or $24 \mathrm{~h}$ ), and the results were both negative.

All five studies compared CHM plus conventional western therapy with conventional western therapy. After analyzing separately according to the study design, the results (see Figure 4) regardless of RCTs or retrospective studies with a control group showed that there was no statistical difference between the experimental and control groups (RR 1.42, 95\% CI 0.76 to 2.62, 1 RCT (Fu et al., 2020b); RR 1.20, 95\% CI 0.98 to $1.48,4$ retrospective studies with a control group (Li et al., 2020; Qu et al., 2020a; Xia et al., 2020; Yang et al., 2020c)).

Aggravation Rate. A total of 14 studies that compared CHM plus conventional western therapy with conventional western therapy reported on this outcome. Of these, two retrospective studies with a control group (Shi et al., 2020a; Yang et al., 2020a) reported that there were no patients who experienced aggravation in either the experimental or control group. Although one study (Yu et al., 2020) reported this outcome in their trial, we did not enrolled the data on this outcome in the statistical analysis due to the inconsistency between the data presented in the table and in the text. After analyzing separately according to the study design of the remaining 11 studies, the results of RCTs or retrospective studies with a control group both showed that CHM plus conventional western therapy was better than conventional western therapy alone in reducing aggravation rate (RR $0.43,95 \%$ CI 0.23 to $0.80,7$ RCTs (Fu et al., 2020a; Wang et al., 2020c; Duan et al., 2020; Qiu et al., 2020; Sun et al., 2020; Ye, 2020; Fu et al., 2020b); RR 0.37, 95\% CI 0.22 to 0.64, 4 retrospective studies with a control group (Chen et al., 2020; Cheng et al., 2020; Li et al., 2020c; Xia et al., 2020)). Figure 5 illustrates the details of these results.

Mortality Rate. Five studies that compared CHM plus conventional western therapy with conventional western therapy reported this outcome. After analyzing separately according to the study design, the results (see Figure 6) regardless of RCTs or retrospective studies with a control group showed that there was no statistical difference between the experimental and control groups (RR 0.45, 95\% CI 0.09 to
2.13, 3 RCTs (Wang et al., 2020c; Ye, 2020; Yu et al., 2020); RR $0.66,95 \%$ CI 0.35 to $1.27,2$ retrospective studies with a control group (Yang et al., 2020c; Xia et al., 2020)).

\section{Secondary Outcomes}

The results on secondary outcomes are shown in Table 3.

The recovery Rate and the Duration of Main Symptoms (Fever, Cough and Fatigue).

a.The recovery rate of main symptoms A total of six studies including 3 RCTs (Ding et al., 2020; Duan et al., 2020; Sun et al., 2020) and 3 retrospective studies with a control group (Chen et al., 2020; Cheng et al., 2020; Yao et al., 2020) reported the recovery rate of main symptoms. All studies compared CHM plus conventional western therapy with conventional western therapy. Of these, the number of studies that reported the recovery rate of fever, cough and fatigue was six (Chen et al., 2020; Cheng et al., 2020; Ding et al., 2020; Duan et al., 2020; Sun et al., 2020; Yao et al., 2020), six (Chen et al., 2020; Cheng et al., 2020; Ding et al., 2020; Duan et al., 2020; Sun et al., 2020; Yao et al., 2020) and five (Chen et al., 2020; Cheng et al., 2020; Duan et al., 2020; Sun et al., 2020; Yao et al., 2020), respectively.

Regarding studies which explored the recovery rate for fever, after analyzing separately according to the study design, although the pooled data of retrospective studies with a control group showed that CHM plus conventional western therapy was better than conventional western therapy alone (RR $1.34,95 \%$ CI 1.13 to 1.58, 3 retrospective studies with a control group), the pooled result of RCTs showed that there was no statistical difference between the experimental and control groups (RR 1.18, 95\% CI 0.91 to $1.54,3$ RCTs, $I^{2}=64 \%$ ).

Regarding studies which investigated the recovery rate of cough, the results of RCTs or retrospective studies with a control group both showed that CHM in combination with conventional western therapy was superior to conventional western therapy alone (RR 1.36, $95 \%$ CI 1.15 to $1.62,3$ RCTs; RR $1.82,95 \%$ CI 1.22 to $2.71,3$ retrospective studies with a control group).

For studies reporting the recovery rate of fatigue following COVID-19, the results regardless of RCTs or retrospective studies with a control group showed that CHM plus conventional western therapy had a higher recovery rate than conventional western 


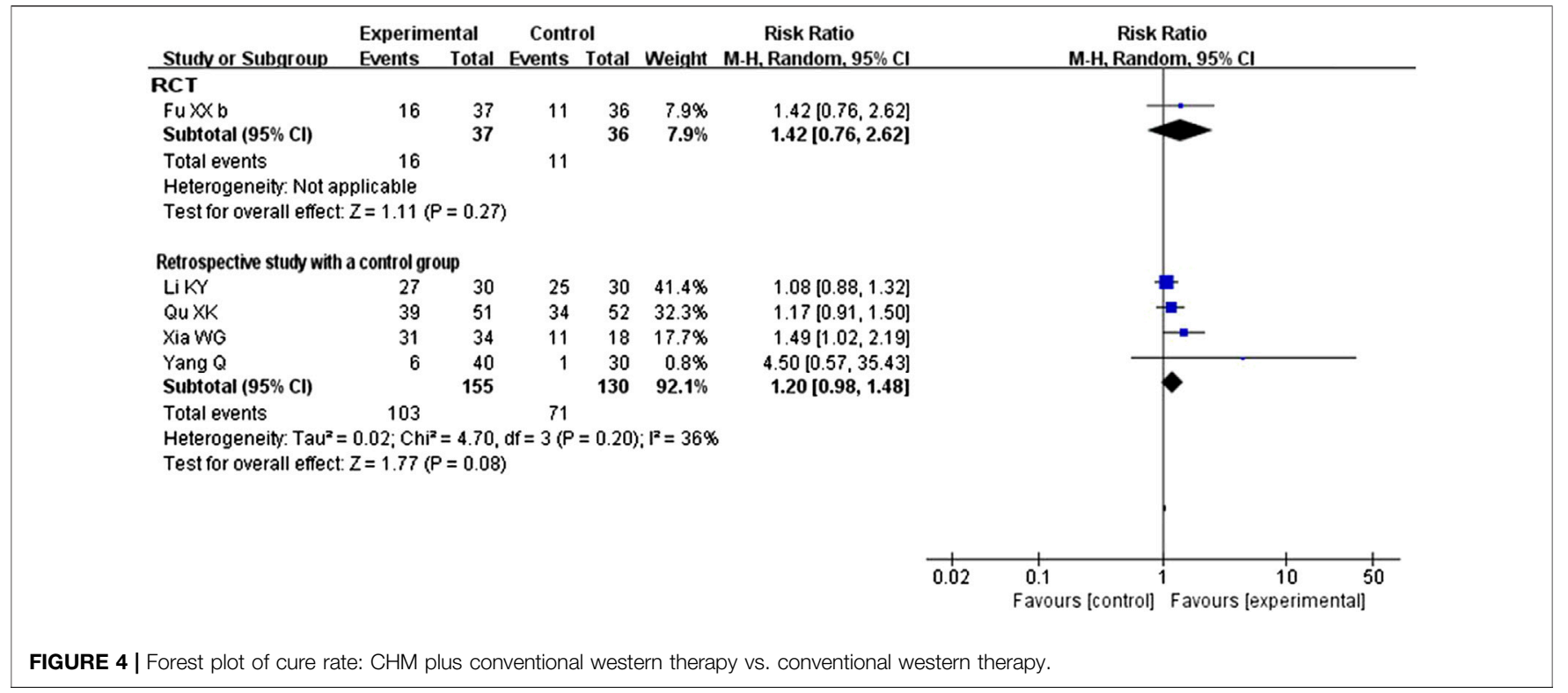

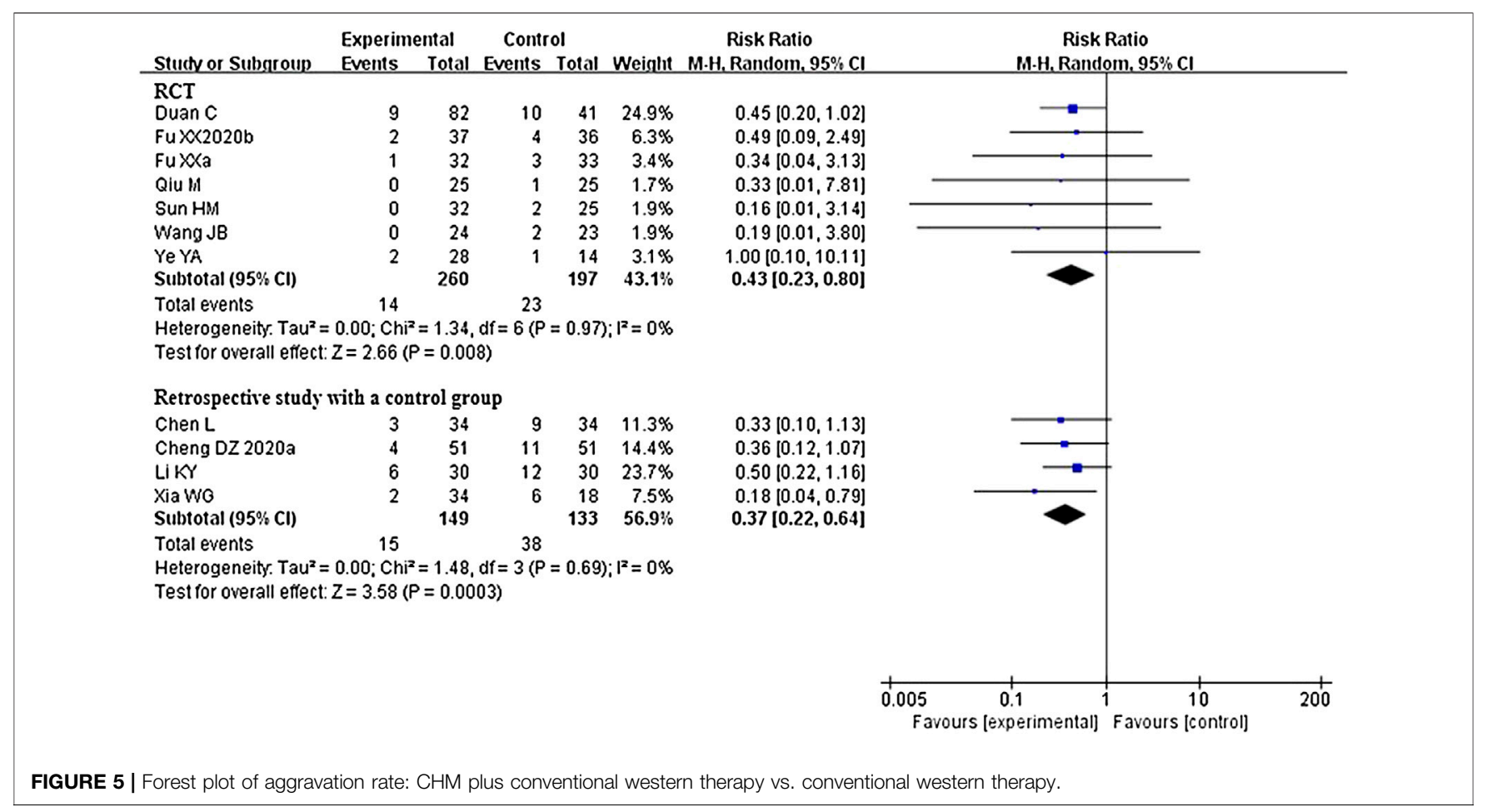

therapy alone (RR 1.33, 95\% CI 1.03 to 1.71, 2 RCTs; RR 1.48, 95\% CI 1.14 to $1.93,3$ retrospective studies with a control group).

b. The duration (time to recovery) of main symptoms A total of 11 studies including 4 RCTs (Zhang et al., 2020a; Wang et al., 2020c; Qiu et al., 2020; Sun et al., 2020), 1 non-RCT (Xiao et al., 2020) and 6 retrospective studies with a control group (Qu et al., 2020a; Chen et al., 2020; Cheng et al., 2020; Li et al., 2020c; Xia et al., 2020; Yao et al., 2020) reported the duration of main symptoms and all of them compared CHM plus conventional western therapy with conventional western therapy. Of these, the number of studies that reported the duration of fever, cough and fatigue was ten (Qu et al., 2020a; Zhang et al., 2020a; Chen et al., 2020; Cheng et al., 2020; Li et al., 2020c; Wang et al., 2020c; Qiu et al., 2020; Xia et al., 2020; Xiao et al., 2020; Yao et al., 2020), eight (Qu et al., 2020a; Zhang et al., 2020a; Chen et al., 2020; Cheng et al., 2020; Li et al., 2020c; Qiu et al., 2020; Xiao et al., 2020; Sun et al., 2020) and five (Qu et al., 2020a; Zhang et al., 2020a; Chen et al., 2020; Cheng et al., 2020; Xiao et al., 2020), respectively. 
TABLE 2 | Chinese herbal medicine used twice or more frequently.

The name of Chinese herbal medicine (CHM)

Frequency (N)

Percentage (\%)

Type 1 of CHM: Prescribed herbal decoction

Maxing Shigan Tang [麻杏石甘汤]

Dayuanyin [达原饮]

Qingfei Paidu Tang [清肺排毒汤]

Xiaochaihu Tang [小柴胡汤]

Ganlu Xiaodu Dan [甘露消毒丹]

Liujunzi Tang [六君子汤]

Sanren Tang [三仁汤]

Feiyan No.1 Fang [肺炎1号方]

Xiaoqinglong Tang [小青龙汤]

Wulingsan [五苓散]

Type 2 of CHM: Oral Chinese patent medicine

Lianhua Qingwen granule/capsule [连花清墭颗粒/胶囊]

Shufeng Jiedu gapsule[疏风解毒胶囊]

Toujie Quwen granule [透解袿瘟颗粒]

Jinhua Qinggan granule [金花清感颗粒]

Shuanghuanglian oral liquid [双黄连口服液]

Type 3 of CHM: Chinese herbal medicine injection

Xuebijing injection [血必净注射剂]

Xiyanping injection [喜炎平注射液]

Tanreqing injection [痰热清注射液]

Shenfu injection [参附注射液]

Shengmai injection [生脉注射液]

Note: Frequency refers to the number of included studies using the CHM. Such as, the frequency of Maxing Shigan Tang is 9, which means that nine included studies used Maxing Shigan Tang.

Percentage $=(\mathrm{N} / 58) * 100 \%$

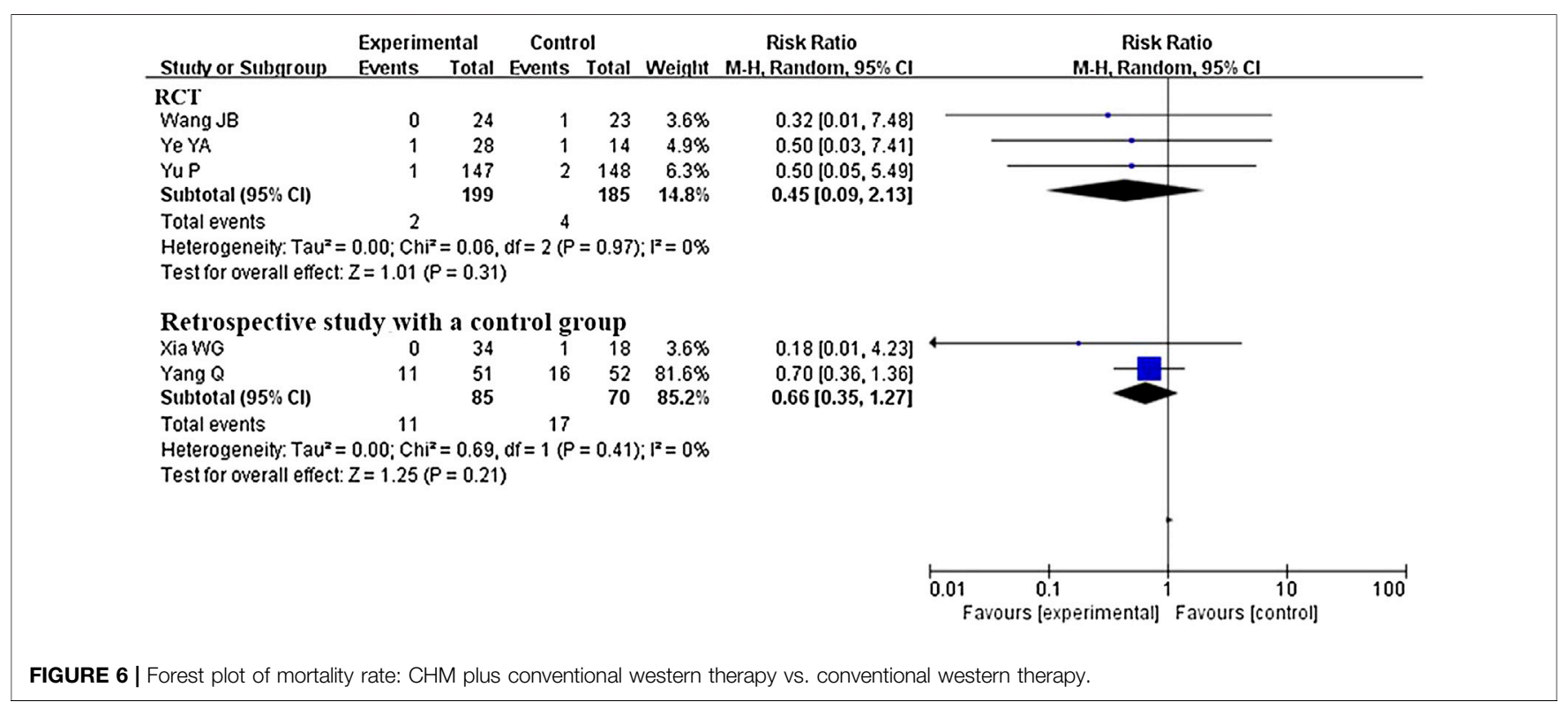

For the duration of fever, one study (Wang et al., 2020c) reported that, the CHM group exhibited a significant improvement in time to fever resolution $(p=0.035)$ compared with the control group. After analyzing separately in light of the other nine studies' design, the results regardless of RCTs, non-RCT or retrospective studies with a control group showed that CHM plus conventional western therapy was better than conventional western therapy alone in shortening the duration of fever (MD-2.08 days, 95\% CI-2.90 to-1.26, 2 RCTs, $I^{2}=$ $60 \%$; MD- 0.83 days, $95 \%$ CI-1.22 to-0.44, 1 non-RCT; MD-1.54 days, 95\% CI-1.82 to-1.26, 6 retrospective studies with a control group).

In shortening the duration of cough, one trial (Sun et al., 2020) reported that $\mathrm{CHM}$ group was superior to conventional western therapy alone in shortening the duration of cough $(\mathrm{P}<0.5)$. After analyzing separately based on the studys' design, the results 
TABLE 3 | The pooled results of secondary outcomes of CHM used with or without conventional western therapy for COVID-19.

\begin{tabular}{|c|c|c|c|c|}
\hline Comparisons and outcomes & Design of the included study & $\begin{array}{l}\text { Number of } \\
\text { study }\end{array}$ & $\begin{array}{l}\text { Number of } \\
\text { participant }\end{array}$ & The pooled results \\
\hline \multicolumn{5}{|l|}{ Chinese herbal medicine + conventional western therapy vs. conventional western therapy } \\
\hline \multirow[t]{2}{*}{ - The recovery rate of fever } & $\mathrm{RCT}$ & 3 & 207 & RR $1.18,95 \% \mathrm{Cl} 0.91$ to $1.54, l^{2}=64 \%$ \\
\hline & $\begin{array}{l}\text { Retrospective study with a control } \\
\text { group }\end{array}$ & 3 & 163 & $\mathrm{RR} 1.34,95 \% \mathrm{Cl} 1.13$ to 1.58 \\
\hline \multirow[t]{2}{*}{ - The recovery rate of cough } & RCT & 3 & 231 & RR $1.36,95 \% \mathrm{Cl} 1.15$ to 1.62 \\
\hline & $\begin{array}{l}\text { Retrospective study with a control } \\
\text { group }\end{array}$ & 3 & 156 & $\mathrm{RR} 1.82,95 \% \mathrm{Cl} 1.22$ to 2.71 \\
\hline \multirow[t]{2}{*}{ - The recovery rate of fatigue } & $\mathrm{RCT}$ & 2 & 108 & $\mathrm{RR} 1.33,95 \% \mathrm{Cl} 1.03$ to 1.71 \\
\hline & $\begin{array}{l}\text { Retrospective study with a control } \\
\text { group }\end{array}$ & 3 & 126 & $\mathrm{RR} 1.48,95 \% \mathrm{Cl} 1.14$ to 1.93 \\
\hline \multirow[t]{3}{*}{ - The duration of fever } & $\mathrm{RCT}$ & 2 & 95 & $\begin{array}{l}\mathrm{MD}-2.08 \text { days, } 95 \% \mathrm{Cl}-2.90 \text { to }-1.26, I^{2} \\
=60 \%\end{array}$ \\
\hline & Non-RCT & 1 & 200 & MD -0.83 days, $95 \% \mathrm{Cl}-1.22$ to -0.44 \\
\hline & $\begin{array}{l}\text { Retrospective study with a control } \\
\text { group }\end{array}$ & 6 & 322 & $\mathrm{MD}-1.54$ days, $95 \% \mathrm{Cl}-1.82$ to -1.26 \\
\hline \multirow[t]{3}{*}{ - The duration of cough } & $\mathrm{RCT}$ & 2 & 95 & $\begin{array}{l}\mathrm{MD}-2.34 \text { days, } 95 \% \mathrm{Cl}-3.32 \text { to }-1.37, I^{2} \\
=56 \%\end{array}$ \\
\hline & Non-RCT & 1 & 200 & MD 0.28 days, $95 \% \mathrm{Cl}-0.40$ to 0.96 \\
\hline & $\begin{array}{l}\text { Retrospective study with a control } \\
\text { group }\end{array}$ & 4 & 214 & $\mathrm{MD}-1.68$ days, $95 \% \mathrm{Cl}-1.92$ to -1.43 \\
\hline \multirow[t]{3}{*}{ - The duration of fatigue } & RCT & 1 & 45 & $\mathrm{MD}-2.35$ days, $95 \% \mathrm{Cl}-2.91$ to -1.79 \\
\hline & Non-RCT & 1 & 200 & MD -0.33 days, $95 \% \mathrm{Cl}-0.78$ to 0.12 \\
\hline & $\begin{array}{l}\text { Retrospective study with a control } \\
\text { group }\end{array}$ & 3 & 136 & $\mathrm{MD}-1.75$ days, $95 \% \mathrm{Cl}-2.01$ to -1.49 \\
\hline - Negative conversion rate of nucleic acid test & $\begin{array}{l}\text { Retrospective study with a control } \\
\text { group }\end{array}$ & 3 & 163 & $\mathrm{RR} 1.32,95 \% \mathrm{Cl} 1.05$ to 1.66 \\
\hline \multirow[t]{3}{*}{ - The improvement rate of chest CT manifestations } & $\mathrm{RCT}$ & 6 & 607 & $\mathrm{RR} 1.28,95 \% \mathrm{Cl} 1.10$ to 1.49 \\
\hline & Non-RCT & 1 & 200 & RR $1.21,95 \% \mathrm{Cl} 1.05$ to 1.40 \\
\hline & $\begin{array}{l}\text { Retrospective study with a control } \\
\text { group }\end{array}$ & 7 & 484 & RR $1.22,95 \% \mathrm{Cl} 1.03$ to $1.45, l^{2}=60 \%$ \\
\hline \multirow[t]{2}{*}{ - The recovery rate of chest CT manifestations } & $\mathrm{RCT}$ & 2 & 355 & RR $1.42,95 \% \mathrm{Cl} 1.00$ to 2.02 \\
\hline & $\begin{array}{l}\text { Retrospective study with a control } \\
\text { group }\end{array}$ & 3 & 251 & $\mathrm{RR} 1.50,95 \% \mathrm{Cl} 0.97$ to 2.31 \\
\hline $\begin{array}{l}\text { - The time from receiving treatment to the beginning of chest CT manifestations } \\
\text { improvement }\end{array}$ & $\begin{array}{l}\text { Retrospective study with a control } \\
\text { group }\end{array}$ & 2 & 140 & $\mathrm{MD}-2.23$ days, $95 \% \mathrm{Cl}-2.46$ to -2.00 \\
\hline - Length of hospitalization & $\begin{array}{l}\text { Retrospective study with a control } \\
\text { group }\end{array}$ & 4 & 290 & $\begin{array}{l}\text { MD }-0.42 \text { days, } 95 \% \mathrm{Cl}-3.49 \text { to } 2.64, I^{2} \\
=95 \%\end{array}$ \\
\hline \multirow[t]{3}{*}{ - Adverse events } & $\mathrm{RCT}$ & 3 & 270 & $\mathrm{RR} 2.06,95 \% \mathrm{Cl} 0.34$ to 12.38 \\
\hline & Non-RCT & 1 & 200 & RR $1.00,95 \% \mathrm{Cl} 0.21$ to 4.84 \\
\hline & $\begin{array}{l}\text { Retrospective study with a control } \\
\text { group }\end{array}$ & 4 & 276 & RR $0.87,95 \% \mathrm{Cl} 0.26$ to 2.93 \\
\hline Chinese herbal medicine vs. conventional western therapy & & None & & \\
\hline
\end{tabular}


regardless of RCTs or retrospective studies with a control group showed that CHM plus conventional western therapy was superior to conventional western therapy alone (MD-2.34 days, 95\% CI-3.32 to-1.37, 2 RCTs, $I^{2}=56 \%$; MD-1.68 days, $95 \%$ CI-1.92 to-1.43, 4 retrospective studies with a control group). However, the results from one non-RCT showed that there was no statistical difference between the experimental and control groups (MD 0.28 days, $95 \%$ CI -0.40 to $0.96,1$ non-RCT).

Regarding those studies reporting the duration of fatigue as secondary outcome, both RCTs and retrospective studies with a control group showed better effects for the CHM plus conventional western therapy when compared with conventional western therapy alone (MD -2.35 days, 95\% CI-2.91 to-1.79, 1 RCT; MD-1.75 days, 95\% CI-2.01 to-1.49, 3 retrospective studies with a control group). However, the result from one non-RCT showed that there was no statistical difference between the two groups (MD-0.33 days, $95 \%$ CI- 0.78 to $0.12,1$ non-RCT).

Negative Conversion Rate of Nucleic Acid Test for SARS-Cov-19. A total of three retrospective studies with a control group $(\mathrm{Qu}$ et al., 2020a; Yang et al., 2020a; Zhang et al., 2020b) reported this outcome and all compared CHM plus conventional western therapy with conventional western therapy. Pooled data from 3 studies showed that $\mathrm{CHM}$ in combination with conventional western therapy was superior to conventional western therapy alone (RR 1.32, 95\% CI 1.05 to 1.66 ) in improving the negative conversion rate of nucleic acid test for SARS-Cov-19.

Improvement or Recovery of Chest CT Manifestations. A total of 16 studies (Yu et al., 2020; Sun et al., 2020; Fu et al., 2020a; Ding et al., 2020; Xiao et al., 2020; Cheng et al., 2020; Liu et al., 2020c; Zhang et al., 2020b; Li et al., 2020c; Yang et al., 2020c; Xia, et al., 2020; Shi et al., 2020a; Yang et al., 2020a; Qiu et al., 2020; Zhang et al., 2020a; Chen et al., 2020) reported this outcome and all compared CHM plus conventional western therapy with conventional western therapy.

Of these, 14 studies (Fu et al., 2020a; Shi et al., 2020a; Yang et al., 2020a; Zhang et al., 2020a; Zhang et al., 2020b; Chen et al., 2020; Cheng et al., 2020; Yang et al., 2020c; Ding et al., 2020; Qiu et al., 2020; Sun et al., 2020; Xia et al., 2020; Xiao et al., 2020; Yu et al., 2020) the improvement rate of chest CT manifestations (improvement rate = the number of patients with improvement of chest CT manifestations/the total number of patients in experimental or control group $\times 100 \%)$. After analyzing separately according to the study design, the results regardless of RCTs, non-RCT or retrospective studies with a control group showed that CHM plus conventional western therapy was better than conventional western therapy alone in increasing the improvement rate of chest CT manifestations (RR1.28, 95\% CI 1.10 to $1.49,6$ RCTs; RR 1.21, 95\% CI 1.05 to $1.40,1$ non-RCT; RR $1.22,95 \%$ CI 1.03 to $1.45,7$ retrospective studies with a control group). Five studies (Fu et al., 2020a; Yu et al., 2020; Chen et al., 2020; Yang et al., 2020c, Liu et al., 2020c) reported the recovery rate of chest CT manifestations (recovery rate $=$ the number of patients with recovery of chest CT manifestations / the total number of patients in experimental or control group $\times 100 \%$ ). After analyzing separately according to the studys' design, the results demonstrated that there was no statistical difference between the two groups in increasing the recovery rate of chest CT manifestations (RR 1.42, 95\% CI 1.00 to 2.02, 2 RCTs; RR $1.50,95 \%$ CI 0.97 to $2.31,3$ retrospective studies with a control group).

The other two retrospective studies with a control group (Li et al., 2020c; Liu et al., 2020c) reported the time from receiving the treatment to the beginning of chest CT manifestations improvement and the pooled analysis from the two studies showed that CHM plus conventional western therapy was superior to conventional western therapy alone in shortening the time (MD-2.23 days, 95\% CI-2.46 to -2.00 , two retrospective studies with a control group).

Length of Hospitalization. A total of four retrospective studies with a control group (Shi et al., 2020a; Yang et al., 2020c; Qiu et al., 2020; Xia et al., 2020) reported length of time in hospital as an outcome. All four studies compared CHM plus conventional western therapy with conventional western therapy. The pooled analysis from the four studies showed that there was no statistical difference between the experimental and control groups (MD -0.42 days, $95 \%$ CI -3.49 to $2.64, I^{2}=95 \%$ ) in shortening the length of hospitalization.

Adverse Events. A total of 16 studies reported this outcome and all compared CHM plus conventional western therapy with conventional western therapy. Of these, eight studies (Fu et al., 2020a; Yang et al., 2020a; Zhang et al., 2020a; Fu et al., 2020b; Chen et al., 2020; Liu et al., 2020c; Xia et al., 2020; Yu et al., 2020) reported that no adverse events occurred in either the experimental or control group. Pooled data from the other eight studies (Qu et al., 2020a; Li et al., 2020c; Wang et al., 2020c; Yang et al., 2020c; Zhang et al., 2020c; Ding et al., 2020; Duan et al., 2020; Xiao et al., 2020) showed that there was no statistical difference between the experimental and control groups (RR 2.06, 95\% CI 0.34 to 12.38, three RCTs (Duan et al., 2020; Ding et al., 2020; Wang, et al., 2020c); RR 1.00, 95\% CI 0.21 to 4.84 , one non-RCT (Xiao et al., 2020); RR 0.87, 95\% CI 0.26 to 2.93, four retrospective studies with a control group (Zhang et al., 2020a; Li et al., 2020c; Yang, et al., 2020c; Qu et al., 2020a)). The adverse events reported in these eight studies were mild abdominal pain, diarrhea, nausea, vomiting and drug allergy, et al.

\section{Subgroup Analysis}

As all controlled studies compared CHM plus conventional western therapy with conventional western therapy, we failed to perform the subgroup analysis based on the use of CHM with or without conventional western therapy. Therefore, we only conducted the subgroup analysis based on the level of severity of COVID-19 (non-serious, serious or a mix of non-serious and serious) for primary outcomes.

With regard to cure rate, although a pooled data of five studies that reported this outcome showed that CHM plus conventional western therapy was superior to conventional western therapy in improving it (RR 1.21, 95\% CI 1.01 to 1.45), the results (see Supplement-Figure 1) of the subgroup analysis based on the level of severity of COVID-19 showed that there was no statistical difference between the experimental and control groups (RR 1.69, 95\% CI 0.72 to 3.92, two studies (Qu et al., 2020a; Fu et al., 2020b) involving 143 non- 
serious patients; RR 1.17, 95\% CI 0.91 to 1.50 , one study (Yang et al., 2020c) involving 103 serious patients; RR 1.23, 95\% CI 0.87 to 1.72 , two studies (Li et al., 2020c; Xia et al., 2020) involving 112 patients, a mix of non-serious and serious, $I^{2}=62 \%$ ).

Regarding aggravation rate, a total of 11 studies (Fu et al., 2020b; Duan et al., 2020; Sun et al., 2020; Fu et al., 2020a; Ye, 2020; Cheng et al., 2020; Li, et al., 2020c; Xia et al., 2020; Qiu et al., 2020; Chen et al., 2020; Wang et al., 2020c) that reported this outcome were used to conduct meta-analysis, and the results (see Supplement Figure-2) from the 11 studies showed that CHM plus conventional western therapy was better than conventional western therapy alone in reducing aggravation rate ( $\mathrm{RR} 0.40,95 \% \mathrm{CI} 0.26$ to 0.59 ). Of which, seven studies (Duan et al., 2020; Sun et al., 2020; Fu et al., 2020a; Cheng et al., 2020, Qiu et al.,2020 ; Chen et al., 2020; Wang et al., 2020c) included only patients with non-serious COVID-19, and pooled data from the seven studies showed that $\mathrm{CHM}$ plus conventional western therapy was better than conventional western therapy (RR $0.37,95 \%$ CI 0.22 to 0.63 , seven studies). One study (Ye, 2020) included only patients with serious COVID19 , the results showed that there was no statistical difference between the experimental and control groups (RR 1.00, 95\% CI 0.10 to 10.11 , one study). The remaining three studies (Fu et al., 2020b; Li et al., 2020c; Xia et al., 2020) included both non-serious patients and serious patients with COVID-19, and the results from the three studies showed a lower aggravation rate in the experimental group compared with the control group (RR $0.40,95 \%$ CI 0.21 to 0.79 , three studies).

For mortality rate, a total of five studies (Wang et al., 2020c; Yang et al., 2020c; Xia et al., 2020; Ye, 2020; Yu et al., 2020) were included, and pooled data from five studies showed that there was no statistical difference between the experimental and control groups (RR 0.62, 95\% CI 0.34 to 1.14 ) in reducing mortality rate. The results (see Supplement-Figure 3) of the subgroup analysis based on the level of severity of COVID-19 showed that there was also no statistical difference between the two groups (RR 0.43, 95\% CI 0.06 to 2.86, two study (Wang et al., 2020c; Yu et al., 2020) involving 342 non-serious patients; RR 0.69, 95\% CI 0.36 to 1.31, two studies (Yang et al., 2020c; Ye, 2020) involving 145 serious patients; RR 0.18 , 95\% CI 0.01 to 4.23 , one study (Xia et al., 2020) involving 52 patients, a mix of nonserious and serious).

\section{Analysis of Case Series and Case Reports}

A total of 12 case series and 24 case reports were included in our review. Of which, one case series and 7 case reports involving 111 patients only used CHM, and 11 case series and 19 case reports involving 828 patients used $\mathrm{CHM}$ plus conventional western therapy. The authors of the 36 articles concluded that CHM with or without conventional western therapy was beneficial for the treatment of COVID-19.

With regard to 111 patients who received CHM treatment for a period of time from 4 to 11 days, one case series and one case report involving 100 patients reported that 42 patients were cured (42/100), 7 case reports involving 13 patients reported that 13 patients were negative for nucleic acid test (13/13), one case series and 6 case reports involving 54 patients reported that 30 patients with the recovery of fever (30/54), one case series and one case report involving 71 patients reported that 17 patients with the recovery of cough (17/71), one case series involving 75 patients reported that 20 patients with the recovery of fatigue (20/75), one case series and 5 case reports involving 96 patients reported that 87 patients (87/96) showed improvement or recovery of chest CT manifestations.

For 828 patients who received CHM plus conventional western therapy for a period of time from 6 to 15 days, 4 case series and 6 case reports involving 641 patients reported that 561 patients were cured (561/641), 6 case series and 16 case reports involving 182 patients reported that 179 patients were negative for nucleic acid test (179/ 182), 5 case series and 13 case reports involving 271 patients reported that 258 patients with the recovery of fever (258/271), 5 case series and 3 case reports involving 437 patients reported that 284 patients with the recovery of cough (284/437), 5 case series and 2 case reports involving 327 patients reported that 212 patients with the recovery of fatigue (212/327), and 3 case series and 11 case reports involving 525 patients reported that 483 patients $(483 / 525)$ showed improvement or recovery of chest CT manifestations. In addition, there were 3 case series which reported adverse events. Of these, 2 case series reported that no adverse events occurred, and the remaining reported that seven patients with the treatment of CHM plus conventional western therapy experienced adverse events including vomiting (4), dizziness 2) and rash (1).

\section{DISCUSSION}

Although RCT is the gold standard to evaluate the therapeutic effects of interventions, it cannot answer all the important questions about a given intervention (Black, 1996). Considering the characteristics of sudden acute infectious diseases and the practical problems of ethics and informed consent, the implementation of RCT faces more challenges under conventional medical conditions (Yang et al., 2020b). Many questions in medical research are investigated in observational studies having a role in research into the benefits and harms of medical interventions (Black, 1996; Glasziou et al., 2004), having an important reference for the preliminary evaluation of the therapeutic effects of CHM and clinical decision-making. In this case, other types of studies (e.g., non-RCT, retrospective studies, case-series) were included in our review.

\section{Summary of the Main Findings}

A total of 58 clinical studies whose purpose were to evaluate the therapeutic effects of CHM used with or without conventional western therapy for COVID-19 were included. The included studies involved RCTs, non-RCT, retrospective studies with a control group, case-series and case-reports. In total the studies involved 2773 COVID-19 patients, 1921 (69.28\%) of them received CHM. The severity of COVID-19 varied from nonserious (mild and common) and serious (severe and critical). Most of the studies used a combination of $\mathrm{CHM}$ and conventional western therapy. Analysis of the frequency of different $\mathrm{CHM}$ indicated that the most frequently used oral Chinese patent medicine, Chinese herbal medicine injection and prescribed herbal decoction were Lianhua Qingwen granule/capsule, Xuebijing injection, and Maxing Shigan Tang, respectively.

This review suggested that CHM in combination with conventional western therapy appeared better than conventional western therapy alone in reducing aggravation rate, increasing the 
recovery rate or shortening the duration of main symptoms (fever, cough and fatigue), improving the negative conversion rate of nucleic acid test, increasing the improvement rate of chest CT manifestations and shortening the time from receiving the treatment to the beginning of chest CT manifestations improvement. For the primary outcomes, subgroup analyses were conducted based on the level of severity of COVID-19 and suggested that CHM in combination with conventional western therapy had more significant effect than conventional western therapy in reducing aggravation rate for non-serious patients.

In terms of reducing mortality rate and shortening the length of hospitalization, there was no statistical difference between the CHM combined conventional western therapy group and the conventional western therapy group. Although some studies have reported adverse events (e.g., mild abdominal pain, diarrhea, nausea and vomiting) in the CHM plus conventional western therapy group, but there was also no statistical difference between the experimental and control groups. This suggests that the use of CHM did not increase the risk of adverse events.

Although in this review there were no pooled results for $\mathrm{CHM}$ used alone from controlled studies for COVID-19, one case-series and seven case-reports that were included reported that $\mathrm{CHM}$ alone may play a positive therapeutic role in the treatment of COVID-19.

\section{Strengths and Limitations}

This review systematically collected the evidence from clinical studies whose purpose was to evaluate the therapeutic effects and safety of CHM with or without conventional western therapy for COVID-19. Relevant clinical studies were analyzed from the aspects of general characteristics, quality assessment, analysis of the use of CHM, therapeutic effects and safety of CHM for COVID-19 patients, providing important evidence for future related research.

However, this review did not summarize the specific administration methods of $\mathrm{CHM}$ in all the included studies, especially considering the complexity of prescribed herbal decoction use, which may require further specific research in the future. Therefore, this review cannot be directly used to guide clinical practice. In addition, all included studies were conducted in China, whether this evidence is equally applicable to other countries outside China needs further international study.

\section{Implications for Further Research}

The benefits for the use of CHM for COVID-19 needs to be verified by more rigorous designed and implemented clinical trials, especially randomized controlled trials. The following points should be noted when conducting relevant RCTs: 1) Clear reporting of random allocation and random concealment; 2) Application of blinding to participants, personnel (doctors), outcome evaluators and statistical analysts; 3) Design and register the study protocol; 4) Definition of important outcomes, such as time to cure, aggravation and mortality; 5) Selection of CHM: considering the difficulty in the use of herbal decoction (e.g., dosage of herbal medicien, especially about its use outside China), we suggest that trials of oral Chinese patent medicine or Chinese herbal medicine injection should be given priority to verify the therapeutic effects and safety of these two, so as to find safe, effective and convenient medications to cure more COVID-19 patients as soon as possible. Unfortunately, in our this research, we did not to perform subgroup analysis on oral Chinese patent medicine, Chinese herbal medicine injection and prescribed Chinese herbal medicine decoction.

\section{CONCLUSION}

Current low certainty evidence suggests that there maybe a tendency that CHM plus conventional western therapy is superior to conventional western therapy alone. The use of $\mathrm{CHM}$ did not increase the risk of adverse events.

\section{AUTHOR CONTRIBUTIONS}

J-PL and S-BL conceived and designed the review. S-BL, Y-YZ, CS, Y-QL, B-YL, C-HL, and Z-YT were responsible for the searching, screening and selecting studies. S-BL, Y-YZ, Y-QL, CS, B-YL, ND, YJ, X-WZ, C-HL, Y-PZ, and MX participated in data extraction. S-BL and Y-QL assessed the risk of bias of the included trials. S-BL performed the statistical analysis. Y-YZ, Y-QL, B-YL, and ND helped to perform the statistical analysis. S-BL drafted the manuscript. J-PL, YZ, NR, CS, and Y-YZ were all involved in critically revising the manuscript. All authors have read and approved the final manuscript. All authors approved the final version of the article, including the authorship list.

\section{FUNDING}

J-PL is supported by the National Natural Science Foundation project (No. 81830115) in China. NR (visiting Professor of Beijing University of Chinese Medicine) is funded by Overseas Expertise Project, Ministry of Education of China (No. G20190001122).

\section{ACKNOWLEDGMENTS}

We greatly thank Ming Yang from Centre for Evidence-Based Chinese Medicine, Beijing University of Chinese Medicine, for the suggestion during the review protocol development process.

\section{SUPPLEMENTARY MATERIAL}

The Supplementary Material for this article can be found online at: https://www.frontiersin.org/articles/10.3389/fphar.2020.583450/ full\#supplementary-material. 


\section{REFERENCES}

Ba, Y. M., Wang, L. Q., Li, W. N., Li, M., Tao, R., Zuo, X. H., et al. (2020). Multi-center clinical study on 451 cases of COVID-19 treated with 'Pneumonia No.1 Formula'. World Chin. Med. 55(13), 1962-1066. doi:10.3969/j.issn.1673-7202.2020.13.021

Black, N. (1996). Why we need observational studies to evaluate the effectiveness of health care. BMJ 312 (7040), 1215-1218. doi:10.1136/bmj.312.7040.1215

Chandan, S., Milon, M., Muhammad, T. I., Miquel, M., Anca, O. D., Alfred, M., et al. (2020). Potential therapeutic options for COVID-19: current status, challenges, and future perspectives. Front. Pharmacol. 11, 572870. doi:10. 3389/fphar.2020.572870

Chen, L., Liu, F., Wu, J. H., Song, H. Y., Xia, J. S., Sheng, B., et al. (2020). Clinical efficacy of Shufeng Jiedu Capsule combined with western medicine in treatment of common COVID-19 patients by retrospective analysis. Chin. J. Exp. Tradit. Med. Form. 26 (16), 14-20. doi:10.13422/j.cnki.syfjx.20201628

Cheng, D. Z., and Li, Y. (2020). Clinical effectiveness and case analysis in 54 NCP patients treated with Lanhuaqingwen granules. World Chin. Med. 15, 150-154. doi:10.3969/j.issn.1673-7202.2020.02.006

Cheng, D. Z., Wang, W. J., Li, Y., Wu, X. D., Zhou, B., and Song, Q. Y. (2020). Curative effects of Chinese medicine Lianhua Qingwen on 51 cases of novel coronavirus pneumonia patients: a multi-center retrospective study. Tianjin J. Tradit. Chin. Med. 37, 509-516. doi:10.11656/j.issn.1672-1519.2020.05.06

Deng, Z., Hu, Y. X., Yang, P., Zheng, P., Peng, W. F., Ren, B. Q., et al. (2020). Diagnosis and treatment of an critical pneumonia patient with COVID-19: case report. J. Med. Virol. 92 (10), 1728-1730. doi:10.1002/jmv.25802

Ding, X. J., Zhang, Y., He, D. C., Zhang, M. Y., Tan, Y. J., and Yu, A. R. (2020). Clinical effect and mechanism of Qingfei Touxie Fuzheng Recipe in the treatment of novel coronavirus pneumonia. Her. Med. 39, 640-644. doi:10. 3870/j.issn.1004-0781.2020.05.012

Dong, L., Li, Y. Q., Yang, S. J., Liu, M. N., and Liu, J. L. (2020). 1 case syndrome differentiation and treatment of new coronavirus pneumonia. Pharmacol. Clin. Chin. Mater. Clin. Med. 36, 69-71. doi:10.13412/j.cnki.zyyl.20200312.001

Duan, C., Xia, W. G., Zheng, C. J., Sun, G. B., Li, Z. L., et al. (2020). Clinical observation of Jinhua Qinggan granule in treating novel coronavirus pneumonia. J. Tradit. Chin. Med., 1-5.

Feng, Q. M., Li, X. Q., She, Z., Zhao, F. C., Li, W. Y., Li, H. T., et al. (2020). Study on method of "three protections" in damp pestilence and treatment of COVID-19 based on harmonic method. Shanghai J. Tradit. Chin. Med. 54, 41-45. doi:10. 16305/j.1007-1334.2020.06.091

Fu, X. X., Lin, L. P., and Tan, X. H. (2020). 2 cases reports of corona virus disease 2019 treated by Toujie quwen granules. J. Jinan Univ. 41, 152-156. doi:10. 11778/j.jdxb.2020.02.009

Fu, X. X., Lin, L. P., and Tan, X. H. (2020a). Clinical study on treatment of cases of COVID-19 with Toujie Quwen granules. Chin. J. Exp. Tradit. Med. Form. 26, 44-48. doi:10.13422/j.cnki.syfjx.20201314

Fu, X. X., Lin, L. P., and Tan, X. H. (2020b). Clinical study on 37 case of COVID-19 treated with integrated traditional Chinese and western medicine. Tradit. Chin. Drug Res. Pharmacol. 31, 600-604. doi:10.19378/j.issn.1003-9783.2020.05.016

Gao, X. S., Zhang, Y. L., and Han, L. H. (2020). Clinical observation of Jinyinhua oral liquid in the treatment of one patient with common type of coronavirus disease 2019. China Pharm. 29, 58-59. doi:10.3969/j.issn.1006-4931.2020. 07.016

Glasziou, P., Vandenbroucke, J. P., and Chalmers, I. (2004). Assessing the quality of research. BMJ 328 (7430), 39-41. doi:10.1136/bmj.328.7430.39

He, Q., Ye, X. X., and Xu, B. (2020). Two Cases of novel coronavirus pneumonia treated by integrated traditional Chinese and western medicine. Chin. J. Integr. Tradit. West. Med. 40, 378-379. doi:10.7661/j.cjim.20200216.276

Higgins, J. P., Altman, D. G., Gøtzsche, P. C., Jüni, P., Moher, D., Oxman, A. D., et al. (2011). The Cochrane Collaboration's tool for assessing risk of bias in randomized trials. BMJ 18, 343. doi:10.1136/bmj.d5928

Hu, M. L., Dong, R. L., Chen, G., Dong, H., Zhang, M. M., Lu, F. E., et al. (2020). A case report of severe novel coronavirus pneumonia treated with integrated traditional Chinese and western medicine. Chin. J. Integr. Tradit. West. Med. 40, 228-230. doi:10.7661/j.cjim.20200204.065

Huang, L. J., Chen, F. C., Jiang, X. Q., Li, Z. H., Wang, W., and Liu, Y. W. (2020). Clinical characteristics and therapy of novel corona virus pneumonia: 71 cases retrospective analysis. Central South Pharm. 18(5), 739-742. doi:10.7539/j.issn. 1672-2981.2020.05.005

Jiang, Y. (2011). Textual research on the concept of Yi Bing in Chinese medicine. Chin. J. Basic Med. Tradit. Chin. Med. 17, 1060-1062.

Lai, Y. G., Fan, H. J., Hu, Y. J., Fu, Y. C., Yang, F. H., Zhang, W., et al. (2020). Examples of modified Maxing Ganshi decoction for treatment of novel coronavirus pneumonia. Chin. J. Inf. Tradit. Chin. Med. 1-3. doi:10.13190/j. cnki.1005-5304.202002478

Li, D. F., Chen, Y., Li, Y., and Fang, J. (2020a). Treatment of 21 cases of COVID-19 by modified Dayuan decoction: a report of 2 cases. Jiangsu J. Tradit. Chin. Med. 52, 59-61. doi:10.19844/j.cnki.1672-397X.2020.00.014

Li, G. W., Li, K. L., Guo, M. Y., Sun, H. Y., Mao, J. Y., and Liu, X. Q. (2020b). Treatment of novel coronavirus pneumonia based on syndrome differentiation in 2 cases of positive nucleic acid retest. Tianjin J. Tradit. Chin. Med. 37(08), 854-857. doi:10.11656/j.issn.1672-1519.2020.08.04

Li, K. Y., An, W., Xia, F., Chen, M., Yang, P., Liao, Y. L., et al. (2020c). Observation on clinical effect of modified Qingfei Paidu Decoction in treatment of COVID19. Chin. Tradit. Herb. Drugs 51, 2046-2049. doi:10.7501/j.issn.0253-2670. 2020.08.008

Li, S. Y., Li, G. Y., Zhang, H. R., Li, B., Lewis, A. H., and Ci, Z. H. (2020d). Clinical efficacy and experiences of Lung-toxin dispelling formula No.1 treating patients of corona virus disease 2019 of severe/critical. Chin. J. Exp. Tradit. Med. Form. 26, 13-20. doi:10.13422/j.cnki.syfjx.20200843

Li, W. N., Ba, Y. M., Tao, R., Zhou, S. S., Yu, B. B., Zhu, X. Y., et al. (2020e). Pneumonia No. 1" in the treatment of severe coronavirus pneumonia: a case report. Hubei J. Tradit. Chin. Med. 42, 3-8.

Li, X. H., Li, L., Zhang, J. Y., Yang, H. S., Wang, X. J., and Gou, C. Y. (2020f). Two cases of common novel coronavirus pneumonia treated by tcm syndrome differentiation. J. Tradit. Chin. Med. 61, 935-937. doi:10.13288/j.11-2166/r. 2020.11 .003

Lin, J. Z., Lan, X. H., and Wang, C. J. (2020). A case of the treatment of novel coronavirus pneumonia based on the combination of syndrome differentiation of wei qi ying blood and syndrome differentiation of viscera. Tianjin J. Tradit. Chin. Med. 37, 251-254. doi:10.11656/j.issn.1672-1519.2020.03.04

Liu, M. J., Tao, Y., Wan, P., and Luo, A. (2020a). Clinical observation of Xue's Fuyang Zhushi decoction in treating 36 cases of novel coronavirus pneumonia with difficulty in transferring nucleic acid into negative. J. Southwest Univ. 42, 1-5. doi:10.13718/j.cnki.xdzk.2020.05.004

Liu, Y., Ren, X., Sun, Y., Yang, C. X., and Xu, Q. (2020b). Diagnosis and treatment of novel coronavirus pneumonia in pregnancy with gastrointestinal symptoms as first manifestations. J. Jilin Univ. 46, 408-412. doi:10.13481/j.1671-587x. 20200234

Liu, Z. L., Li, X. H., Gou, C. Y., Li, L., Luo, X. L., Zhang, C., et al. (2020c). Clinical observation and evaluation of Jinhua Qinggan granules in the treatment of novel coronavirus pneumonia. J. Tradit. Chin. Med., 1-16. doi:10.19852/j.cnki. jtcm.20200409.001

Luo, H., Tang, Q. L., Shang, Y. X., Liang, S. B., and YangLiu, M. J. P. (2020). Can Chinese medicine Be used for prevention of corona virus disease 2019 (COVID19)? A review of historical classics, research evidence and current prevention programs. Chin. J. Integr. Med. 26 (4), 243-250. doi:10.1007/s11655-0203192-6

National Health Commission of the People's Republic of China (2020a). Diagnosis and treatment protocol for novel coronavirus pneumonia. 7th Edn. Available at: http://www.nhc.gov.cn/yzygj/s7653p/202003/46c9294a7dfe4cef80dc7f5912eb1989. shtml (Accessed June 12, 2020).

National Health Commission of the People's Republic of China (2020b). Notice on diagnosis and treatment protocol for novel coronavirus pneumonia. 3rd Edn. Available at: http://www.nhc.gov.cn/xcs/yqfkdt/202001/f492c9153ea9437bb587ce2ffcbeelfa shtml (Accessed June 12, 2020).

Ni, L., Zhou, L., Zhou, M., Zhao, J. P., and Wang, D. W. (2020). Combination of western medicine and Chinese traditional patent medicine in treating a family case of COVID-19 in Wuhan. Front. Med. 14, 210-214. doi:10.1007/s11684020-0757-x

Qiu, M., Li, Q. T., Zhu, D. P., Wang, C. H., Sun, Q. Z., Qian, C. F., et al. (2020). Efficacy observation of maxing xuanfei Jiedu decoction on common type of NCP. J. Emerg. Tradit. Chin. Med. 29(07), 1129-1130+1132. doi:10.3969/j.issn. 1004-745X.2020.07.001 
Qu, X. K., Hao, S. L., Ma, J. H., Wei, G. Y., Song, K. Y., Tang, C., et al. (2020a). Observation on clinical effect of Shufeng Jiedu capsule combined with arbidol hydrochloride capsule in treatment of COVID-19. Chin. Tradit. Herb. Drugs 51, 1167-1170. doi:10.7501/j.issn.0253-2670.2020.05.011

Qu, Y. F., Fang, W., Jin, Y. Z., Qin, C., Niu, X. C., Zhang, N., et al. (2020b). Forty cases of common COVID-19 treated with Modified Ephedra and Apricot Kernel and Gypsum and Licorice decoction combined with western medicine routine treatment. Henan Tradit. Chin. Med. 40, 666-669. doi:10.16367/j.issn. 1003-5028.2020.05.0167

Shi, J., Yang, Z. G., Ye, C., Chen, S. S., Lu, Y. F., Lv, Y., et al. (2020a). Clinical observation on 49 cases of non-critical COVID-19 in Shanghai treated by integrated traditional Chinese and western medicine. Shanghai J. Tradit. Chin. Med. 54, 30-35. doi:10.16305/j. 1007-1334.2020.04.095

Shi, L., Wang, T., Fang, Y. K., and Li, X. H. (2020b). Analysis and thinking of COVID-19 treated by traditional Chinese medicine. Jilin J. Chin. Med. 40, 565-570. doi:10.13463/j.cnki.jlzyy.2020.07.001

Shi, T. F., Zhou, G. C., Zhang, L. Y., Niu, F., Ke, Y. C., Zhou, T., et al. (2020c). Clinical efficacy of Xuanfei Huazhu decoction on 40 cases of COVID-19. Chin. J. Exp. Tradit. Med. Form. 1-6. doi:10.16305/j.1007-1334.2020.04.095

Sun, H. M., Xu, F., Zhang, L., Wei, C., Chen, J. Y., Wang, Q. X., et al. (2020). Study on clinical efficacy of Lianhua Qingke granule in treatment of mild and ordinary COVID-19. Chin. J. Exp. Tradit. Med. Form. 26, 29-34. doi:10. 13422/j.cnki.syfjx.20201438

Tian, Z. H., Wu, B., Xiang, J. J., Ge, J., Qin, K. L., Li, Y. Y., et al. (2020). Theoretical analysis and clinical practice of differentiation and treatment of the novel coronavirus pneumonia by integration of traditional and western medicine. World Chin. Med. 15, 519-523. doi:10.3969/j.issn.1673-7202.2020.04.008

Torequl, I. M., Nasiruddin, M., Khan, I. N., Siddhartha, K. M., Md, K., Thoufiqul, A. R., et al. (2020). A perspective on emerging therapeutic interventions for COVID-19. Front Public Health 8, 281. doi:10.3389/fpubh.2020.00281

Wang, H. F., Zhang, Y. Q., Li, B., Zhu, M. J., Li, S. Y., Ren, W. H., et al. (2020a). Effect of integrated traditional Chinese and western medicine treatment on immune function of 2 patients with severe novel coronavirus pneumonia. Chin. J. Integr. Tradit West. Med. 4, 1. doi:10.7661/j.cjim.20200323.145

Wang, H., Kong, Y. Q., and Wang, M. (2020b). Application of insampaedok-san in the treatment of novel coronavirus pneumonia. J. Emerg. Tradit. Chin. Med., 1-3. doi:10.3969/j.issn.1004-745X.2020.07.006

Wang, J. B., Wang, Z. X., Jing, J., Zhao, P., Dong, J. H., Zhou, Y. F., et al. (2020c). Exploring an integrative therapy for treating COVID-19: a randomized controlled trial. Chin. J. Integr. Med. 26 (9), 648-655. doi:10.1007/s11655020-3426-7

Wang, R. Q., Yang, S. J., Xie, C. G., Shen, Q. L., Li, M. Q., Lei, Q., et al. (2020d). Clinical observation of Qingfeipaidu decoction in treating COVID-19. Pharmacol. Clin. Chin. Mater. Clin. Med. 36, 13-18. doi:10.13412/j.cnki.zyyl. 20200303.002

Wang, Y. C., Tian, X. T., Zhou, R. J., Liu, W., Liu, G. S., and Tian, J. (2020e). Applying the clinical experience of Liu Huimin using Artemisia annua in treating two cases of fever caused by novel coronavirus pneumonia. Clin. J. Chin. Med. 1-3.

Wang, Z. W., Chen, X. R., Lu, Y. F., Chen, F. F., and Zhang, W. (2020f). Clinical characteristics and therapeutic procedure for four cases with 2019 novel coronavirus pneumonia receiving combined Chinese and western medicine treatment. Biosci. Trends. 14, 64-68. doi:10.5582/bst.2020.01030

World Health Organization (2020b). WHO Director-General's opening remarks at the media briefing on COVID-19 on 11 March 2020. Available at: https://www. who.int/dg/speeches/detail/who-director-general-s-opening-remarks-at-the-mediabriefing-on-covid-19-11-march-2020 (Accessed June 12, 2020).

World Health Organization (2020a). WHO Director-General's remarks at the media briefing on 2019-nCoV on 11 February 2020. Available at: https://www. who.int/dg/speeches/detail/who-director-general-s-remarks-at-the-mediabriefing-on-2019-ncov-on-11-february-2020 (Accessed June 12, 2020 ).

Xia, W. G., An, C. Q., Zheng, C. J., Zhang, J. X., Huang, M., Wang, Y., et al. (2020). Clinical observation on 34 patients with novel coronavirus pneumonia (COVID19) treated with intergrated traditional Chinese and western medicine. J. Tradit. Chin. Med. 61, 375-382. doi:10.13288/j.11-2166/r.2020.05.002

Xiao, Q., Jiang, Y. J., Wu, S. S., Wang, Y., An, Jun., Xu, W. P., et al. (2020). Analysis of the value of Chinese medicine Shufeng Jiedu capsule combined with Arbidol in the treatment of mild novel coronavirus pneumonia. J. Emerg. Tradit. Chin. Med. 29, 756-758. doi:10.3969/j.issn.1004-745X.2020.05.002

Xie, Y. F., Ruan, Y. D., and Liu, X. R. (2020a). Clinical observation on 8 novel coronavirus pneumonia cases in Dongguan area treated by integrated traditional Chinese and western medicine. Clin. J. Tradit. Chin. Med. 32(07), 1204-1208. doi:10.16448/j.cjtcm.2020.0703

Xie, Y. F., Ruan, Y. D., and Liu, X. R. (2020b). Clinical summary of 27 cases of common novel coronavirus in Dongguan area treated by combination of traditional Chinese and western medicine. Pract. Tradit. Chin. Intern. Med. 34(9), 5-8+123. doi:10.13729/j.issn.1671-7813.Z20200244

$\mathrm{Xu}$, J. C., Pan, H. T., and Ling, Z. (2020). A case report of curing COVID-2019 with early intervention of traditional Chinese medicine. Clin. J. Traditi. Chin. Med. 32(8), 1416-1419. doi:10.16448/j.cjtcm.2020.0804

Yang, H. M., and Niu, J. H. (2020). One case on the treatment of sever novel coronavirus pneumonia with TCM syndrome differentiation and symptomatic intervention of western medicine. Jiangsu J. Tradit. Chin. Med. 52, 30-34. doi:10.19844/j.cnki.1672-397X.2020.00.011

Yang, M. B., Dang, S. S., Huang, S., Li, Y. J., and Guo, Y. L. (2020a). Multi-center clinical observation of Reyanning mixture in treatment of novel coronavirus pneumonia. Chin. J. Exp. Tradit. Med. Form. 26, 7-12. doi:10.13422/j.cnki.syfjx.20201321

Yang, M., Zhang, Y., Dong, F., Zhang, Y. Y., Gong, Y. Y., Fei, Y. T., et al. (2020b). Thoughts on clinical research strategies and methods of Chinese medicine participating treatment of COVID-19. Chin. J. Integr. Tradit. West. Med. 40 (03), 283-286. doi:10.7661/j.cjim. 20200216.275

Yang, Q., Sun, Q. G., Jiang, B., Xu, H. J., Luo, M., Xie, P., et al. (2020c). Retrospective clinical study on treatment of COVID-19 patients with integrated traditional Chinese and western medicine. Chin. Tradit. Herb. Drugs 51, 2050-2054. doi:10.7501/j.issn.0253-2670.2020.08.009

Yao, K. T., Liu, M. Y., Li, X., Huang, J. H., and Cai, H. B. (2020). Retrospective clinical analysis on treatment of coronavirus disease 2019 with traditional Chinese medicine Lianhua Qingwen. Chin. J. Exp. Tradit. Med. Form. 26, 8-12. doi:10.13422/j.cnki.syfjx.20201099

Ye, Y. A. (2020). Guideline-based Chinese herbal medicine treatment plus standard care for severe coronavirus disease 2019 (G-CHAMPS): evidence from China. Front. Med. 7, 256. doi:10.3389/fmed.2020.00256

Yu, P., Li, Y. Z., Wan, S, B., and Wang, Y. (2020). Observation of therapeutic effects of Lianhua Qingwen granule combined with Arbidol on mild novel coronavirus pneumonia. Chin. Pharmaceut. J. 55(12), 1042-1045. doi:10.11669/cpj.2020.12.014

Zhang, C. T., Yang, Y., You, F. M., Huang, Q. S., Gao, P. Y., Tang, J. Y., et al. (2020a). Clinical study on COVID-19 from the perspective of "yidujiashi" theory. Pharmacol. Clin. Chin. Mater. Clin. Med. 36, 43-45.

Zhang, C. Y., Zhang, S., Wang, W., and Jiang, X. Q. (2020b). Clinical observation of Xuebijing in the treatment of COVID-19. Chin. J. Hosp. Pharm. 40(9), 964-967. doi:10.13286/j.1001-5213.2020.09.03

Zhang, Y., Xie, Y. Z., Wang, F., Jin, J. X., and Han, L. Y. (2020c). A clinical study and experience on diagnosis and treatment of 24 cases with COVID-19 based on “damp-heat epidemic virus". J. Cap. Univ. Med. Sci. 41, 277-282. doi:10.3969/j. issn.1006-7795.2020.02.023

Zhao, D. K., Cai, H. Y., Luo, W., Huang, Y. X., Ma, H., Chen, Y. Y., et al. (2020). One case of COVID-19 treated by integrated traditional Chinese and western Medicine. Jilin J. Chin. Med. 40, 561-564. doi:10.13463/j.cnki. jlzyy.2020.05.001

Zhou, Y. J., Yu, J. H., Guo, J. C., Bao, J. F., Chen, H. P., Huang, J. S., et al. (2020), Clinical observation of 40 cases of novel coronavirus pneumonia with cold and dampness obstructive pulmonary syndrome treated by 'Pneumonia No.2 Formula'. Zhejiang J. Integr. Tradit. Chin. West. Med. 30, 263-266.

Conflict of Interest: The authors declare that the research was conducted in the absence of any commercial or financial relationships that could be construed as a potential conflict of interest.

Copyright $\odot 2021$ Liang, Zhang, Shen, Liang, Lai, Dai, Li, Tian, Zhang, Jiang, Xiong, Zhang, Zhang, Robinson and Liu. This is an open-access article distributed under the terms of the Creative Commons Attribution License (CC BY). The use, distribution or reproduction in other forums is permitted, provided the original author $(s)$ and the copyright owner(s) are credited and that the original publication in this journal is cited, in accordance with accepted academic practice. No use, distribution or reproduction is permitted which does not comply with these terms. 


\section{APPENDIX 1 \\ SEARCH STRATEGIES FOR THE NINE ELECTRONIC DATABASES AND CLINICAL TRIAL REGISTRATION PLATFORMS (CTRP).}

\begin{tabular}{|c|c|c|}
\hline Databases/CTRP & Search strategy & Time limit \\
\hline CNKI & $\begin{array}{l}\text { Since CNKI has set up a thematic platform for COVID - 19, the "treatment" section of the platform was selected } \\
\text { for manual retrieval }\end{array}$ & As of April 30, 2020 \\
\hline VIP & $\begin{array}{l}\text { \#1: M = Xinxing Guangzhuang Bingdu Bing(新型冠状病毒病) OR Xinguan Feiyan (新冠肺炎) OR } 2019 \\
\text { Guanzhuang Bingdu (2019冠状病毒病) OR COVID-19 OR 2019-nCOV OR NCP } \\
\text { \#2: M = Zhongyi (中医) ORZhongyao (中药) OR Caoyao (草药) OR Tangji (汤剂) ORZhongchengyao (中成药) OR } \\
\text { Zhusheji (注射剂) OR Zhongxiyi Jiehe (中西医结合) } \\
\text { \#3: \#1 AND \#2 }\end{array}$ & $\begin{array}{l}\text { From January } 1 \text { to April 30, } \\
2020\end{array}$ \\
\hline Wanfang & $\begin{array}{l}\text { \#1: Major Topic: "Xinxing Guangzhuang Bingdu Bing (新型冠状病毒病)" + "Xinguan Feiyan (新冠肺炎)" + " } 2019 \\
\text { Guanzhuang Bingdu Bing (2019冠状病毒病)" + "COVID-19" + "2019-nCOV" + "NCP" } \\
\text { \#2: Major Topic: "Zhongyi (中医)" + "Zhongyao (中药)" + "Caoyao (草药)" + "Tangji (汤剂)" + "Zhongchengyao (中 } \\
\text { 成药)" + "Zhusheji (注射剂)" + "Zhongxiyi Jiehe (中西医结合)" } \\
\text { \#3: \#1 AND \#2 }\end{array}$ & $\begin{array}{l}\text { From January } 1 \text { to April 30, } \\
2020\end{array}$ \\
\hline SinoMed & $\begin{array}{l}\text { \#1: ("Xinxing Guangzhuang Bingdu Bing (新型冠状病毒病)"[标题:智能] OR "Xinguan Feiyan (新冠肺炎)"[标题:智 } \\
\text { 能] OR "2019 Guanzhuang Bingdu Bing (2019冠状病毒病)"[标题:智能] OR "COVID-19"[标题:智能] OR } \\
\text { "2019-nCOV"[标题:智能] OR "NCP"[标题:智能]) } \\
\text { \#2: ("Zhongyi (中医)"[标题:智能] OR "Zhongyao (中药)"[标题:智能] OR "Caoyao (草药)"[标题:智能] OR "Tangji (汤 } \\
\text { 剂)"[标题:智能] OR "Zhongchengyao (中成药)"[标题:智能] OR "Zhusheji (注射剂)"[标题:智能] OR "Zhongxiyi } \\
\text { Jiehe (中西医结合)"[标题:智能]) } \\
\text { \#3: \#1 AND \#2 }\end{array}$ & $\begin{array}{l}\text { From January } 1 \text { to April 30, } \\
2020\end{array}$ \\
\hline PubMed & $\begin{array}{l}\text { (((Corona virus disease-19 OR COVID-19 OR } 2019 \text { novel coronavirus OR 2019-nCOV OR NCP[MeSH Major } \\
\text { Topic]) AND (Chinese medicine OR traditional Chinese medicine OR herbal medicine OR decoction OR patent } \\
\text { medicine OR injection OR integrated Chinese and western medicine[MeSH Major Topic]) AND ("2020/01/ } \\
\text { 01"[Date-Publication] : "2020/04/30"[Date-Publication]) }\end{array}$ & $\begin{array}{l}\text { From January } 1 \text { to April 30, } \\
2020\end{array}$ \\
\hline Emabse & $\begin{array}{l}\text { \#1: ab,ti: corona virus disease-19 OR COVID-19 OR } 2019 \text { novel coronavirus OR 2019-nCOV OR NCP } \\
\text { \#2: ab,ti: Chinese medicine OR traditional Chinese medicine OR herbal medicine OR decoction OR patent } \\
\text { medicine OR injection OR integrated Chinese and western medicine } \\
\text { \#3: \#1 AND \#2 }\end{array}$ & $\begin{array}{l}\text { From January } 1 \text { to April 30, } \\
2020\end{array}$ \\
\hline ChiCTR & Title search was carried out using Xinxing Guangzhuang Bingdu (新型冠状病毒) and COVID-19 as search terms & As of April 30, 2020 \\
\hline ClinicalTrials.gov & Searched in covid-19 special registration section & As of April 30, 2020 \\
\hline $\begin{array}{l}\text { BioRxiv, MedRxiv, } \\
\text { arxiv }\end{array}$ & Title or abstract search was carried out using COVID-19 as search terms & As of April 30, 2020 \\
\hline
\end{tabular}

